Published in final edited form as:

Nat Immunol. 2016 June ; 17(6): 626-635. doi:10.1038/ni.3443.

\title{
Inflammatory triggers associated with exacerbations of COPD orchestrate plasticity of group 2 innate lymphoid cells in the lungs
}

\author{
Jonathan S Silver ${ }^{1}$, Jennifer Kearley ${ }^{1}$, Alan M Copenhaver ${ }^{1}$, Caroline Sanden ${ }^{2,3}$, Michiko \\ Mori $^{2}$, Li Yu ${ }^{4}$, Gretchen Harms Pritchard ${ }^{5}$, Aaron A Berlin ${ }^{1}$, Christopher A Hunter ${ }^{5}$, Russell \\ Bowler $^{6}$, Jonas S Erjefalt ${ }^{2,3}$, Roland Kolbeck ${ }^{1}$, and Alison A Humbles ${ }^{1}$ \\ ${ }^{1}$ Department of Respiratory, Inflammation and Autoimmunity, Medlmmune, Gaithersburg, \\ Maryland, USA \\ ${ }^{2}$ Department of Experimental Medical Science, Lund University, Lund, Sweden \\ ${ }^{3}$ Medetect, Lund, Sweden \\ ${ }^{4}$ Non-Clinical Biostatistics, Department of Translational Sciences, Medlmmune, Gaithersburg, \\ Maryland, USA \\ ${ }^{5}$ University of Pennsylvania, School of Veterinary Medicine, Philadelphia, Pennsylvania, USA \\ ${ }^{6}$ National Jewish Health, Denver, Colorado, USA
}

\begin{abstract}
Innate lymphoid cells (ILCs) are critical mediators of mucosal immunity, and group 1 ILCs (ILC1 cells) and group 3 ILCs (ILC3 cells) have been shown to be functionally plastic. Here we found that group 2 ILCs (ILC2 cells) also exhibited phenotypic plasticity in response to infectious or noxious agents, characterized by substantially lower expression of the transcription factor GATA-3 and a concomitant switch to being ILC1 cells that produced interferon- $\gamma$ (IFN- $\gamma$ ). Interleukin 12 (IL-12) and IL-18 regulated this conversion, and during viral infection, ILC2 cells clustered within inflamed areas and acquired an ILC1-like phenotype. Mechanistically, these ILC1 cells augmented virus-induced inflammation in a manner dependent on the transcription factor T-bet. Notably, IL-12 converted human ILC2 cells into ILC1 cells, and the frequency of ILC1 cells in patients with chronic obstructive pulmonary disease (COPD) correlated with disease severity and
\end{abstract}

\footnotetext{
Reprints and permissions information is available online at http://www.nature.com/reprints/index.html. Correspondence should be addressed to A.A.H. (humblesa@medimmune.com). AUTHOR CONTRIBUTIONS

J.S.S., J.K. and A.A.H. planned all experiments; J.S.S. and J.K. executed and analyzed all experiments; A.M.C., L.Y., G.H.P. and A.A.B. planned and executed specific experiments; C.S., M.M. and J.S.E. cut, stained and analyzed all histology sections and developed the algorithms for analysis of ILC location; L.Y. analyzed and generated the statistical data and graphs for the COPDGene study; G.H.P. and C.A.H. provided $T b \times 21^{-1-}$ mice and reagents; R.B. provided blood samples from patients with COPD and control subjects; C.A.H., J.S.E., R.K. and A.A.H. provided feedback and edits; and J.S.S. and A.A.H. wrote the manuscript.
}

COMPETING FINANCIAL INTERESTS

The authors declare competing financial interests: details are available in the online version of the paper.

Note: Any Supplementary Information and Source Data files are available in the online version of the paper. 
susceptibility to exacerbations. Thus, functional plasticity of ILC2 cells exacerbates anti-viral immunity, which may have adverse consequences in respiratory diseases such as COPD.

ILCs are a population of tissue-resident innate lymphocytes with diverse roles in mucosal inflammation, including defense against pathogens, maintenance of epithelial barrier function, containment of commensal microbiota, tissue repair and regulation of metabolism ${ }^{1,2}$. Despite their limited numbers, ILCs have a substantial effect on ongoing immune responses and are classified into functionally discrete subsets, ILC1, ILC2 and ILC3, remarkably similar to the $\mathrm{CD}^{+}{ }^{+}$helper $\mathrm{T}$ cell lineages ${ }^{3}$. Many of the transcription factors and cytokines that govern $\mathrm{CD}^{+}$helper $\mathrm{T}$ cell subsets also have critical roles in the corresponding ILC groups. Thus, the transcription factor T-bet is critical for ILC1 development and activity, while the transcription factors GATA-3 and ROR $\gamma \mathrm{t}$ regulate ILC2 function and ILC3 function, respectively ${ }^{4-15}$.

Emerging evidence indicates that ILC subsets are not 'fixed' and exhibit considerable functional plasticity depending on the inflammatory milieu ${ }^{1,16}$. For example, ILC 3 cells can co-express T-bet and ROR $\gamma$ t, produce IFN- $\gamma$ and differentiate into ILC1 cells in response to a variety of inflammatory factors ${ }^{4,17-22}$. This ability to switch is bi-directional, as human ILC1 cells can convert into ILC 3 cells $^{23}$. Additionally, Toll-like-receptor-2 ligands can promote the production of IL-5 and IL- 13 by ILC 3 cells, which suggests that these cells might be able to differentiate into ILC2 cells ${ }^{24}$. Despite the phenotypic flexibility demonstrated for ILC1 and ILC3 cells, it is not clear whether ILC2 cells exhibit any functional plasticity.

COPD is a disorder associated with long-term exposure to cigarette smoke and is characterized by progressive, irreversible loss of lung function ${ }^{25}$. A subset of patients with COPD experience acute worsening of symptoms following respiratory-tract infection, and these exacerbations are a notable cause of morbidity and mortality ${ }^{26,27}$. Influenza virus, respiratory syncytial virus, rhinovirus and nontypeable Haemophilus influenzae are among the main triggers of exacerbations of $\mathrm{COPD}^{26,28}$; however, little is understood about the susceptibility of these patients to infection. Various cytokines of the IL-1 family, including IL-1, IL-18 and IL-33, are linked to smoke-associated inflammation and infection-induced exacerbations in mouse models ${ }^{29-31}$.

Exposure to cigarette smoke 'silences' ILC2 function, and this is associated with an exaggerated type 1 anti-viral response ${ }^{31}$. Thus, changes in lung-resident ILC populations have a substantial effect on the inflammatory response, although the relationships between the local ILC subsets in these models have remained unclear. Here we found that lungresident ILC2 cells significantly downregulated their expression of the IL-33 receptor (ST2) and GATA-3 in response to COPD-associated stimuli, including viruses, bacteria and/or exposure to cigarette smoke. Subsequently, a T-bet ${ }^{+}$IFN- $\gamma^{+}$population of cells emerged with high expression of the receptors for IL-12 (IL-12R) and IL-18 (IL-18R). The tight correlation between the loss of ST2 expression and increase in IL-18R expression suggested that ILC2 cells were directly converting into 'ILC1-like' cells. Indeed, adoptive transfer of ILC2 cells demonstrated these cells were switching to an ILC1 phenotype in response to viral challenge. Further, when visualized in lung tissue, ILCs undergoing phenotypic 
changes were clustered in areas of virus-associated inflammation, near myeloid cells producing IL-12 and IL-18. This phenotypic switch was biologically relevant, since ILC1 cells induced an exaggerated, T-bet-dependent response to viral infection. Moreover, human ILC2 cells upregulated their expression of ILC1 markers in response to IL-12, and patients with COPD had significantly elevated frequencies of circulating ILC1 cells that correlated strongly with disease progression and exacerbation history. Collectively, these data demonstrate that ILC2 cells exhibit considerable functional plasticity that is dependent on local IL-12 and IL-18 signals, which promote the differentiation of ILC2 cells into ILC1 cells.

\section{RESULTS}

\section{Downregulation of GATA-3 by ILCs after influenza virus}

Cigarette smoke 'silences' lung-resident ILC2 cell responses by suppressing their production of IL-5 and IL-13 (ref. 31), and we assessed the relevance of this change and whether other COPD-associated stimuli could do the same. Lung ILC2 cells were defined as a population of non-T cells, non-natural killer (NK) cells that lacked expression of lineage markers $\left(\mathrm{Lin}^{-}\right)$and had high expression of the CD90 (Thy-1), the cytokine receptor IL-7Ra, CD44, ICOS, GATA-3 and ST2 (Fig. 1a,b). Although there were small populations of ILC1 and ILC 3 cells in the lungs, $85-90 \%$ of the total lung ILC pool was ILC2 cells, defined as GATA-3 ${ }^{\text {hi }}$ (Fig. 1c). Since ST2 expression is dynamically regulated following exposure to cigarette smoke $^{31}$, we developed a reporter mouse that expressed ST2 tagged with green fluorescent protein (GFP) (Supplementary Fig. 1a,b). In these mice, ILC2s were readily identifiable as $\mathrm{Lin}^{-} \mathrm{ST} 2-\mathrm{GFP}^{+}$cells (Fig. 1d and Supplementary Fig. 1b) and were confirmed as the predominant ILC subset (Fig. 1c,d). Interestingly, infection with influenza A virus resulted in a rapid loss of GATA-3 expression relative to that of ILC2 cells from uninfected mice; this was observed through $6 \mathrm{~d}$ after infection (Fig. 1e). Furthermore, loss of GATA-3 expression correlated with a substantial reduction in the expression of ST2 in these cells (Fig. 1f), which was not associated with egress or death of ILC2 cells (Supplementary Fig. $1 \mathrm{c}-\mathrm{f})$.

We next assessed expression of receptors for the virus-associated type 1 cytokines IL-12 and IL-18 (ref. 32) on ILCs. Strikingly, the loss of ST2 on ILC2 cells strongly correlated with a parallel increase in the frequency of the IL-18Ra ${ }^{+}$ILCs (Fig. 1f,g). Expression of IL-12R $\beta 2$ did not correlate with the loss of ST2 (data not shown); however, there was significantly higher expression of this receptor on the ILC pool in infected mice than in that of uninfected mice (Fig. 1h). T-bet is an ILC1 marker that promotes $\mathrm{T}_{\mathrm{H}} 1$-like responses 33 , and the loss of GATA-3 in ILC2 cells occurred concomitantly with an increase in the frequency of T-bet ${ }^{\text {hi }}$ ILCs (Fig. 1i). Indeed, virus-induced T-bet ${ }^{+}$ILCs were in the IL-18Ra ${ }^{+}$subset (Fig. 1j) and had lower expression of the cytokine receptor CD25 (IL-2Ra), IL-7Ra, ICOS and the stemcell-factor receptor c-Kit (CD117) than that of ST2 ${ }^{\text {hi }}$ ILCs (Supplementary Fig. 1g). Further, the majority of these cells were positive for the proliferation marker Ki67 (Fig. 1j,k and Supplementary Fig. 1h).

Consistent with the phenotypic changes noted above, ILCs from influenza-virus-infected mice produced much less IL-5 and IL-13 and substantially more IFN- $\gamma$ than did ILCs from 
mock-treated mice ex vivo (Fig. 11 and Supplementary Fig. 1i). Collectively, these data demonstrated a major functional and phenotypic change in lung-resident ILCs during viral challenge characterized by a decrease in ILC2 activity and the expansion of an ILC1 cell population.

\section{Multiple COPD-associated triggers alter ILC populations}

Exacerbations of COPD are associated with viral and bacterial infection ${ }^{2}$. Strikingly, we observed a similar ILC phenotype in response to a strain of influenza A virus (PR8) different from that used above, respiratory syncytial virus, the bacteria Staphylococcus aureus and nontypeable $H$. influenzae: each pathogen induced a loss of GATA-3 expression and a subsequent increase in the expression of IL-12R $\beta 2$, IL-18Ra and T-bet in the lung ILC population (Fig. 2a-f and Supplementary Fig. 2a-f).

That shift in ILC subgroups was not limited to infectious triggers, because exposure to cigarette smoke also induced a substantial loss of GATA-3 expression and the emergence of an IL-12R $\beta 2^{+} \mathrm{IL}^{-18 \mathrm{Ra}^{+}}$T-bet ${ }^{+}$ILC1 population, and both of those characteristics were significantly altered through chronic exposure to smoke (Fig. 2g-m). Furthermore, when exposure to cigarette smoke was combined with viral infection, the changes in ILC phenotype were substantially augmented (Supplementary Fig. 2g-1). These data suggested that the phenotypic switch in the local ILC population was not specific to a particular trigger and was a general response to pathogenic and/or environmental insults.

\section{Derivation of ILC1 cells from the local ST2+ ILC2 cell pool}

The strong negative correlation between the expression of ST2 and that of IL-18Ra on ILCs during infection, coupled with their limited population expansion ${ }^{31}$, indicated that ILC1 cells might emerge from the local ILC2 pool. To investigate this, we expanded lung ILC2 populations with IL-33, purified the cells and cultured them with various cytokines (Supplementary Fig. 3a-c). Strikingly, stimulation with IL-18 alone or co-stimulation with IL-12 and IL-18 induced the production of IFN- $\gamma$ by ILC 2 cells, whereas IL-12 or IL-15 alone had no effect (Fig. 3a,b). Paradoxically, ILC2 cells co-expressed IL-13 and IFN- $\gamma$ following co-stimulation with IL-12 and IL-18 (Supplementary Fig. 3b,c).

ILC responses were assessed in vivo following intranasal administration of IL-12 plus IL-18, IL-33 alone, or a combination of all three, to mice of the SCID ('severe combined immunodeficiency') strain. Co-administration of IL-12 and IL-18 to ILCs resulted in significantly lower expression of GATA-3 and ST2 than that of PBS- or IL-33-treated (control) ILCs (Fig. 3c and Supplementary Fig. 3d,e). Furthermore, treatment with IL-12 plus IL-18 markedly increased the frequency of IL-18Ra ${ }^{+}$T-bet ${ }^{+}$ILCs, which had abundant production of IFN- $\gamma$ but less production of IL- 5 and IL-13 ex vivo than that of ILCs isolated from mice of the other treatment groups (Fig. 3d-g and Supplementary Fig. 3f-i). As expected, IL-33 alone significantly increased the number of IL-5- and IL-13-producing ILC2 cells (Fig. 3e,f and Supplementary Fig. 3h). Co-administration of IL-12 plus IL-18 induced an increase in the total number of ILC1 cells. However, in the presence of IL-33, the ILC1 population expansion and IFN- $\gamma$ production was substantially increased, but at the expense of ILC2 cells (Fig. 3e,g and Supplementary Fig. 3i). These newly induced cells also co- 
expressed IL-5 and IFN- $\gamma$ (Fig. 3h and Supplementary Fig. 3j), consistent with the emergence of ILC1 cells from the local ILC2 pool. Thus, IL-12 and IL-18 co-regulated ILC1 population expansion in vivo, while IL-33 acted in a context-dependent manner to amplify this response.

II1rI1 (which encodes ST2) is a direct target of GATA-3 in ILC2 cells ${ }^{11,34}$; to determine whether ILC1 populations expanded from the local ILC2 pool, we repeated the experiments described above, using the ST2-GFP reporter mice. Comparison of ST2-GFP expression versus IL-18Ra expression revealed two distinct populations of ILCs in naive lungs, ST2GFPhi IL-18Ra ${ }^{-}$ILC2 cells (93.9\%) and ST2-GFP ${ }^{-}$IL-18Ra ${ }^{+}$ILC1 cells (3.13\%), and cotreatment with IL-12, IL-18 and IL-33, resulted in an increased frequency of IL-18Ra ${ }^{+}$ ILC1 cells (Fig. 3i). The expression of ST2-GFP on emerging IL-18Ra ${ }^{+}$cells, although lower than that on naive ILC2 cells, was still significantly greater than that on naive ILC1 cells (Fig. 3i). Furthermore, $\sim 50 \%$ of IL-18Ra ${ }^{+}$cells had intermediate expression of ST2GFP (Fig. 3j). Thus, ILC1 cells were derived from the local ILC2 pool rather than from the outgrowth of existing ILC1 cells in the lungs.

Consistent with that hypothesis, comparison of the gene-expression profiles of ILC2 cells sorted from naive mice and those of ILC2 (ST2 IL-18Ra ${ }^{-}$) cells and ILC1 (ST2 $\left.{ }^{-} \mathrm{IL}-18 \mathrm{Ra}^{+}\right)$ cells sorted from mice treated with IL-12, IL-18 and IL-33 (called 'cytokine-treated mice' here) revealed partially overlapping but distinct molecular patterns. ILC2 cells isolated from the lungs of both naive mice and cytokine-treated mice had high expression of $\mathrm{T}_{\mathrm{H}} 2$ cellassociated transcripts, including Gata3, Rora, II4, II5, I19, I113, Penk, Areg, II17rb and II1r11 (Fig. 3k). In contrast, ILC1 cells from cytokine-treated mice had an altered gene profile relative to that of ILC2 cells from naive or cytokine-treated mice, characterized by lower expression of the aforementioned ILC2-associated transcripts but higher expression of Tbx21, Ifng, II12rb2, II18r1, CXcr3 and Ccr5 mRNA (Fig. 3k). The ILC1 cells from cytokine-treated mice had higher expression of $\mathrm{T}_{\mathrm{H}} 1$ cell-associated transcripts but much lower expression of Prf1, Gzma, Gzmb and Gzmc (which encode cytolytic products), relative to that of naive liver ILC1 cells or lung NK cells (Fig. 3k). There were also differences among ILC subsets of cytokine-treated mice in their expression of mRNA encoding chemokine receptors (for example, $C c r 5, C c 117$ and $C x 3 c r 1$ ) (Fig. 3k). In addition, the expression II7ra, II2r and Icos was lower in activated ILC1 cells than in ILC2 cells (Fig. $3 \mathrm{k}$ ). Thus, plasticity correlated with altered ILC molecular signatures, consistent with proteomic changes observed via flow cytometry (Supplementary Fig. 1g).

\section{Direct ILC2-to-ILC1 conversion during viral challenge}

For direct assessment of the conversion of ILC2 cells, lung ILC2s from reporter mice in which all cells express GFP underwent population expansion with intranasal IL-33 treatment and were sorted by flow cytometry. The resultant $\mathrm{GFP}^{+} \mathrm{ILC} 2$ cells were transferred into host mice deficient in mature lymphocytes ( $\mathrm{Rag}^{-{ }^{--}} \mathrm{II2 \textrm {rg } ^ { - 1 }}$ mice), $12 \mathrm{~h}$ before infecting the host mice with influenza A virus (Supplementary Fig. 4a). GFP ${ }^{+}$ILCs infiltrated the lungs of recipient mice and were readily identifiable by flow cytometry (Fig. 4a,b). At day 7 after infection, $\mathrm{GFP}^{+}$cells had significantly downregulated their GATA-3 expression, and this correlated with striking upregulation of the expression of IL-18Ra and IL-12R $\beta 2$ (Fig. 4c- 
h); similar phenotypic changes were also observed at day 10 after infection (Supplementary

Fig. 4b-i). T-bet expression was undetectable at these time points (data not shown), potentially due to the delayed immune response in these mice. Upon ex vivo stimulation with IL-12 plus IL-18, the $\mathrm{GFP}^{+}$cells were able to produce IFN- $\gamma$, and this change coincided with down-regulation of the production of IL-13 (Supplementary Fig. 4j).

Despite the histologically irregular response to influenza A virus (Fig. 4i), immunohistochemistry (IHC) confirmed a uniform infection rate in the lungs of all mice (Supplementary Fig. 5a). Strikingly, the transferred GFP ${ }^{+}$ILCs (positive and negative controls, Supplementary Fig. 5b,c) seemed to cluster within the virus-associated inflammatory foci (Fig. 4i,j). GFP ${ }^{+}$ILCs were also observed in uninflamed regions of the parenchymal tissue, although these were much fewer in number than those in the inflamed regions and were predominantly solitary (Fig. $4 \mathrm{j}$ ). Double IHC revealed that the clusters of ILCs co-localized with influenza-virus-positive epithelial cells, as well as with perivascular regions neighboring the inflamed airways (Fig. 5a,b). Notably, a combination of IHC and in situ hybridization revealed that myeloid-derived cells expressing $I 112$ and $I 118$ mRNA were frequently identified in close proximity to $\mathrm{GFP}^{+}$ILCs within the inflamed airways (Fig. 5c,d and Supplementary Fig. 5d-h).

To assess and quantify ILC-associated expression of GATA-3 in the lungs, we assigned scores to individual $\mathrm{GFP}^{+}$ILCs on the basis of their staining intensity for the DNA-binding dye DAPI and GATA-3. The transferred GFP ${ }^{+}$ILCs were GATA- $3{ }^{\text {hi }}$ in naive recipient mice, in contrast to ILCs from infected mice (Fig. 5e). 'Scattergram' analysis of individual GFP ${ }^{+}$ cells confirmed that lungs from virus-infected mice contained substantially more GATA-3 $3^{\text {lo }}$ ILCs than did lungs from uninfected mice (Fig. 5f). Normalization of GATA-3 expression in each $\mathrm{GFP}^{+}$cell to that cell's nuclear DAPI content revealed a significantly lower frequency of GATA- ${ }^{\text {hi }}$ ILCs in infected mice than in uninfected (control) mice (Fig. $5 \mathrm{~g}, \mathrm{~h}$ ).

Interestingly, there was a time-dependent loss of GATA-3 expression in ILCs from influenza-virus-treated mice (Fig. 5 f,g), a result we also confirmed by flow cytometry (data not shown).

Due to the patchy, uneven nature of the infection, we assessed whether the loss of GATA-3 expression was specific to the inflamed, cell-dense areas of the lungs (Fig. 5i,j). Focused analysis of the inflamed and uninflamed regions of infected lungs revealed that GATA-3 ${ }^{\text {lo }}$ ILCs (Fig. 5k) were much more frequent within the virus-associated areas (Fig. 5i-k). In contrast, the ILCs found in the surrounding, uninfected tissue regions were predominantly GATA-3 ${ }^{\text {hi }}$ (Fig. 5i-k). In summary, these data demonstrated that ILC2 cells migrated to areas associated with viral replication and pro-inflammatory cytokine expression and directly converted to an ILC1-like phenotype.

\section{Amplification of anti-viral inflammation by ILC1 cells}

We investigated the function of T-bet in the maintenance and/or proliferation of ILC1 cells through the use of T-bet-deficient ( $\mathrm{Tb} \times 2 \mathrm{I}^{-1-}$ ) mice. Interestingly, T-bet was not required for the loss of GATA-3 expression or for the gain of expression of IL-12R 32 or IL-18Ra on ILCs (Fig. 6a-f). However, Tbx $21^{-1-}$ ILCs produced much less IFN- $\gamma$ than wild-type cells did (Fig. 6g). Moreover, ILCs from wild-type and $T b \times 21^{-1-}$ mice responded similarly to 
intranasal administration of IL-12 plus IL-18, although again T-bet was required for maximal expression of IFN- $\gamma$ (Supplementary Fig. 6a-h).

Strategies to specifically deplete mice of ILCs are limited and, as expected ${ }^{35}$, pre-treatment of mice with antibody to CD90 resulted in the depletion of $>90 \%$ of lung-resident ILCs (Supplementary Fig. 6i,j). However, this antibody also eliminated $\sim 70 \%$ of NK cells, as well as a population of lung-resident $\mathrm{CD} 90^{+} \mathrm{CD} 45^{-} \mathrm{CD} 166^{+} \mathrm{Sca}-1^{+}$mesenchymal stem cells (Supplementary Fig. 6i-p). Therefore, to assess the biological relevance of ILC plasticity, we performed adoptive transfer of ILC1 or ILC2 cells. We treated C57BL/6 mice with IL-33 (ILC 2 cells) or with a combination of IL-12, IL-18 and IL-33 (ILC1 cells), then isolated total lung ILCs from those mice and transferred them into $\mathrm{Rag}^{-/-} \mathrm{II2 \textrm {rg } ^ { - / - }}$ mice $12 \mathrm{~h}$ before infecting the host mice with influenza A virus (Supplementary Fig. 6q). Strikingly, mice that received ILC1 cells exhibited significantly greater weight loss following infection than that of mice reconstituted with ILC2 cells (Fig. 6h). Moreover, transfer of ILC1 cells correlated with enhanced production of pro-inflammatory cytokines (Fig. 6i). Differences in cellular inflammation following the transfer of ILC1 cells or ILC2 cells at this time point were not apparent (data not shown), presumably due to the delayed response to infection in $\mathrm{Rag}^{-/-} I 12 \mathrm{rg}^{-/-}$mice. To investigate whether T-bet or IFN- $\gamma$ was required for the inflammatory potential of ILC1 cells, we repeated the adoptive transfer using wild-type or $T b \times 21^{-1-}$ ILC1 cells. Again, the transfer of ILC1 cells resulted in exaggerated production of $\mathrm{T}_{\mathrm{H}} 1$ cytokines that was T-bet dependent (Fig. 6j). Strikingly, the absence of T-bet in the $T b \times 21^{-1-}$ ILC1 cells was associated with significantly greater production of the $\mathrm{T}_{\mathrm{H}} 2$ cytokines IL-4 and IL-5 than that of wild-type ILC1 cells (Fig. 6k). Thus, T-bet promoted the full pro-inflammatory potential of ILC2-derived ILC1 cells during viral challenge.

\section{Induction of plasticity in human ILC2 cells by IL-12}

To determine whether human ILC2 cells can differentiate into ILC1 cells, we sorted $\mathrm{Lin}^{-} \mathrm{IL}-7 \mathrm{Ra}^{+} \mathrm{CRTH} 2{ }^{+} \mathrm{CD} 161^{+}$ILC2 populations from the peripheral blood of healthy donors (Supplementary Fig. 7a,b) and cultured the cells with IL-2 plus IL-33 or with IL-2 plus IL-12 and IL-33, then assessed surface markers and cytokine output. ILC2 cells stimulated with IL-2 plus IL-33 were CRTH2 ${ }^{+}$GATA- $3^{+}$T-bet ${ }^{-} \mathrm{CD} 25^{+} \mathrm{CD} 161^{+} \mathrm{IL}-7 \mathrm{Ra}^{+}(\mathrm{Fig}$. $7 \mathrm{a}-\mathrm{c}$ and Supplementary Fig. 7a-c) and produced large amounts of IL-13 and IL-4 but significantly less IFN- $\gamma$ (Fig. 7d-f). In contrast, the addition of IL-12 to the culture conditions resulted in much lower expression of GATA-3 and CD25 and concomitantly promoted substantially higher T-bet expression in ILC2 cells (Fig. 7a-c). Further, in response to IL-12, human ILC2 cells produced less IL-13 and IL-4 but more IFN- $\gamma$, and the amount of IFN- $\gamma$ was augmented in the presence of IL-33 (Fig. 7d-f). Thus, human ILC2 cells acquired an ILC1 phenotype following stimulation with IL-12 and, analogous to results obtained for mouse ILC2 cells, IL-33 amplified IFN- $\gamma$ production in these cells.

\section{Alteration of ILC populations in COPD}

We assessed the frequency of ILC subsets in the peripheral blood of stable patients in a cohort of the COPDGene study investigating the underlying genetic factors of $\mathrm{COPD}^{36}$. In healthy control subjects, $40 \%$ of circulating ILCs expressed the ILC2 marker CRTH2 (Fig. $8 \mathrm{a}$ ), as well as CD25, IL-7Ra and GATA-3 (data not shown), whereas $\sim 5 \%$ of circulating 
ILCs were ILC1 cells (defined as T-bet ${ }^{\mathrm{hi}}{ }^{11}$ (Fig. 8a and Supplementary Fig. 8a). The frequency of ILC1 cells in the circulation was much higher in patients with COPD than in healthy control subjects, and this correlated with a lower frequency of circulating ILC2 cells (Fig. 8a-c). Moreover, patients with more severe disease (as defined by The Global Initiative for Chronic Obstructive Lung Disease (GOLD) classification III-IV) had a significantly higher frequency of ILC1 cells than that of patients with milder disease (GOLD I-II) (Fig. 8d). Conversely, the frequency of ILC2 cells was much lower in the patients with more severe disease (GOLD III-IV) than in patients with milder disease (GOLD I-II) or healthy smokers (Fig. 8e). Healthy smokers (matched for age and smoking 'pack years') also exhibited significantly higher frequency of ILC1 cells than that of healthy control subjects, although there was an apparent trend for a greater proportion of ILC1 cells in all patients with COPD (GOLD I-IV) than in healthy smokers (Fig. 8b). Furthermore, there was a significant inverse correlation between the frequency of ILC1 cells and lung function, as assessed by FEV1\% predicted ((the FEV1\% of the patient (forced expiratory volume for $1 \mathrm{~s}$ (FEV1) calculated as a percentage of the forced vital capacity (FVC)) divided by the average FEV $1 \%$ of the population for any person of similar age $(R=-0.4162 ; P<0.01)$ and the FEV1/FVC ratio (a calculated ratio used in the diagnosis of COPD; $R=-0.4154 ; P<0.01$ ) (Fig. $8 \mathrm{f}$ and Supplementary Fig. 8b). In contrast, the frequency of ILC2 cells in patients with COPD positively correlated with lung-function parameters (Supplementary Fig. 8c,d). Of note, there was a significant linear trend between the frequency of ILC1 cells and the average number of exacerbations; patients with two or more exacerbations per year had the highest frequency of ILC1 cells and the lowest frequency of ILC2 cells (Fig. 8g,h). Moreover, although the sum of ILC1 cells and the frequency of ILC2 cells remained the same for all groups $(42.3 \% \pm 13.1 \%$ (mean \pm s.d.); Supplementary Fig. 8e), a strong negative correlation between the frequency of ILC1 cells and that of ILC2 cells was observed $(R=-0.5529 ; P<0.001)$ (Fig. 8i), which supported the hypothesis of direct conversion of ILC2 cells into ILC1 cells in COPD. Collectively, these data demonstrated that COPD was associated with substantial changes in the pool of circulating ILCs and that an increase in the frequency of ILC1 cells correlated with disease.

\section{DISCUSSION}

In this report we demonstrated that influenza-virus-induced inflammation was associated with the emergence of ILC1 cells that was the result of their direct conversion from lungresident ILC2 cells. A wide variety of triggers associated with exacerbations of COPD, including infection and exposure to cigarette smoke, initiated this plasticity in ILC2 cells, which suggested that functional conversion is a general response to inflammation. This phenotypic switch involved sequential events: the convergence of ILC2 cells into inflamed areas, significant loss of GATA-3 expression, and exposure to micro-environmental cues, such as IL-12 and IL-18, which dictated the subsequent response.

The transcription factor GATA-3 is the definitive marker for ILC2 cells and has a critical role in their development, function and survival ${ }^{10,11,34}$. Molecular analyses revealed that the emerging ILC1 cells (i.e., formerly ILC2 ('ex-ILC2') cells) shared a gene signature with activated ILC2 cells that was partially overlapping but also distinct, including the coexpression of mRNA encoding GATA-3 and T-bet. Notably, in an accompanying manuscript, 
Ohne et al. confirm a mixed or hybrid $\mathrm{T}_{\mathrm{H}} 1-\mathrm{T}_{\mathrm{H}} 2$ epigenetic signature in 'ex-ILC2 cells' 37 . Indeed, there is emerging evidence for promiscuous transcription-factor expression in ILCs, gradients of which impart unique biological outcomes in these cells. For example, ROR $\gamma \mathrm{t}^{+}$ ILC 3 cells have lower expression of GATA-3 than that of ILC2 cells, yet GATA-3 has a nonredundant role in ILC3 cell homeostasis ${ }^{38,39}$, while expression of T-bet confers distinct functions on gut-resident ILC3 cells in a dose-dependent manner ${ }^{20}$. Notably, the gradient of GATA-3 expression has substantial effects on ILC2 function ${ }^{34,40}$, and in our study here, transferred ILC2 cells exhibited a significant loss in GATA-3 expression and subsequent upregulation of ILC1 markers, including IFN- $\gamma$. Thus, given the role of GATA-3 in maintaining ILC2 fate, loss of this transcription factor alone might be required for functional plasticity.

The striking similarity of the response of lung-resident ILC populations to infectious and noxious agents would suggest that a common biological mechanism underlies these phenotypic changes. IL-12 has emerged as a key regulator of ILC3 plasticity in humans ${ }^{23}$, and this study and the accompanying papers ${ }^{37,41}$ now indicate a direct role for this cytokine in ILC2 plasticity. Indeed, IL-12 is produced during viral and bacterial infections ${ }^{42}$, and intranasal administration of IL-12 and IL-18 resulted in the specific emergence of ILC1 cells. The early expression of IL-12R $\beta 2$ on ILC2 cells before the expression of IL-18Ra and the presence of ILC2 cells that co-expressed ST2 and IL-12R $\beta 2$ (data not shown) were consistent with the hypothesis that IL-12 regulated this phenotypic switch. Furthermore, type I interferons can directly inhibit ILC2 responses $^{43-45}$, which suggests that virusinduced cytokines are critical factors in the conversion of ILC2 cells.

ILCs seem to be sessile and predominantly tissue resident ${ }^{46}$, and the findings we have reported here support this hypothesis. Indeed, the rarity of tissue-resident ILCs, coupled with their limited population expansion ${ }^{31}$, suggests that functional plasticity might be a critical mechanism for ensuring the appropriate inflammatory response. Indeed, extensive IHC analysis revealed that ILCs converged within the inflamed areas of the tissue, in close proximity to myeloid-derived cells expressing IL-12 and IL-18. Such accumulation, together with the substantial cytokine output of ILCs, would suggest these cells can substantially augment micro-environmental cytokine concentrations in response to infection. Notably, ILC1 cells significantly amplified the virus-elicited production of $\mathrm{T}_{\mathrm{H}} 1$ cell-like inflammatory cytokines and weight loss. Remarkably, ILCs associated with the infected regions of the lungs had lower expression of GATA-3 than that of ILCs present in the unaffected areas of the tissue, in support of the idea that ILC2s encountering inflammatory foci are actively directed to switch phenotype. These data highlight the importance of examining local changes, since whole-population-level analysis might obscure or underestimate micro-environmental changes in the phenotype of tissue-resident ILCs.

Long-term exposure to cigarette smoke has been linked to subsequent susceptibility to acute exacerbations of COPD in a subset of patients with this disease ${ }^{47}$, but relatively little is understood about how the immune system responds to this noxious agent. Mechanistically, our report has provided a context for the 'silencing' effect of smoke on ILC2s observed in smoke-exposed mice ${ }^{31}$. The IL-1-family members IL-1, IL-18 and IL-33 augment ILC2 plasticity, and their expression is markedly upregulated during experimental exposure to 
smoke and in patients with $\mathrm{COPD}^{29-31,37,48}$. Thus, respiratory infections might drive hyperinflammatory ILC1 responses in the context of elevated IL-1-related cytokines associated with prior exposure to cigarette smoke.

Published studies have shown that IL-12 can induce upregulation of the expression of ILC1 markers in human ILC3 cells, while ILC1 cells can differentiate into ILC3 cells in the presence of IL-23, IL-1 $\beta$ and retinoic acid ${ }^{4,20,21,23}$. Our data demonstrated that CRTH2 ${ }^{+}$ ILC2s responded to IL-12 by downregulating their expression of GATA-3 and upregulating their expression of T-bet and IFN- $\gamma$, and the two accompanying studies confirm human ILC2 plasticity ${ }^{37,41}$. Moreover, we found that patients with COPD had significantly altered ILC1/ILC2 ratios that correlated with diminished lung function, more-severe disease and susceptibility to acute exacerbations. In their accompanying manuscript, Bal et al. report a similar increase of ILC1 cells in COPD lung tissue ${ }^{41}$. Hence, circulating ILC subtypes reflect the lung ILC populations and might be indicative of disease progression. The strong correlation between the frequency of ILC1 cells and that of ILC2 cells in patients with COPD, echoing the reciprocal relationship between ST2 ${ }^{+}$ILCs and IL-18Ra ${ }^{+}$ILCs in mouse lungs, would suggest that plasticity might be an active process in this disease. Given the phenotypically promiscuous nature of ILCs and the lack of known cell-specific surface markers for their depletion, targeting these cells through manipulation of their plasticity might offer novel therapeutic approaches in the treatment and management of acute exacerbations of COPD.

\section{ONLINE METHODS}

\section{Mice}

BALB/c (Harlan, \#047), SCID (CBySmn.CB17-Prkdcscid/J; Jax, \#001803), C57BL/6 (Jax, \#000664), $\operatorname{Rag}^{-/-} I 12 \mathrm{rg}^{-1-}\left(\right.$ Rag $\left.^{2 \mathrm{~m} 1 \mathrm{Fwa}} I I 2 \mathrm{rg}^{\mathrm{tm} 1 \mathrm{Wjl}}\right)$ deficient (Taconic, \#4111), green fluorescent protein (GFP) transgenic (C57BL/6-Tg(CAG-EGFP)131Osb/LeySopJ, Jax, \#006567) and ST2-GFP reporter mice (C.B6-II1rI Im3548.1(T2a-EGFP)Arte Tg(CAG-Flpe)2

Arte) mice were housed at MedImmune and treated according to protocols approved by the Institutional Animal Care and Use Committee at MedImmune. Tbx 21 deficient (Jax) mice were housed and maintained at the University of Pennsylvania according to institutional guidelines and protocols established by the university. For all mouse experiments, mice were randomized and placed into groups based on weight. Female (age 8-12 weeks at start of experiment) mice were used for any experiments involving only BALB/c, SCID, C57BL/6 or GFP transgenics. For any experiment using $\mathrm{Rag} 2^{-/-} \mathrm{II2 \textrm {rg } ^ { - 1 - }}$ mice, $\mathrm{Tb} \times 2 \mathrm{I}^{-1-}$ mice or ST2GFP reporter mice, 8 - to 12-week-old male and female mice were used and the control mice were sex matched with the group of interest.

\section{Generation of ST2-GFP reporter mice}

A targeting construct was injected into murine blastocysts containing an upstream 4-kb short homology arm followed by a flippase (FLP) recombinase target (FRT)-flanked puromycin resistance cassette nestled in intron 10, then exon 11 of the $I 11$ rl1 gene where the stop codon was deleted and immediately followed by a fusion sequence of the T2A self-cleaving peptide fused to enhanced green fluorescent protein (eGFP) followed by a new stop codon. 
The construct ended with a 6-kb homology arm. All sequences used for the creating the targeting construct were based on the Ensembl transcript ENSMUST00000097772 (Il1rl1-001) which corresponds to Il1rl1 Isoform A (Membrane-bound; Uniprot ID: P14719-1). After Flp-mediated recombination, the puromycin selection gene was deleted, leaving a single FRT site remaining in intron 10 of the targeted Il1rl1 gene followed by the exon11-T2A-eGFP fusion sequence (Supplementary Fig. 1a). All ST2-reporter mice used for experiments were heterozygous $\mathrm{ST} 2^{+/ \mathrm{GFP}}$ mice on a BALB/c background, and littermates were used as controls.

\section{Smoke models}

Mice were exposed to cigarette smoke twice a day, 5 days a week following previously published protocols ${ }^{31}$ and analyzed at time points specified (Fig. 2 and Supplementary Fig. 2).

\section{Bacterial and viral challenge models}

$50 \times \mathrm{TCID}_{50}$ of influenza A virus (A/FM/1/47), 50× $\mathrm{TCID}_{50}$ PR8 strain (both are H1N1) or $10^{6}$ plaque forming units (PFU) of respiratory syncytial virus strain A2 were administered to mice intranasally. In some studies, influenza A virus was administered after smoke exposure according to previously established protocols ${ }^{29,31}$. Mice were analyzed at time points specified in the figures. Mice were treated intranasally with $10^{7}$ colony forming units (CFU) of nontypeable Haemophilis influenzae and analyzed at day 2 and day 5 post-infection, or $10^{5} \mathrm{CFU}$ of Staphylococcus aureus and analyzed at day 5 post-infection.

\section{In situ ILC population expansion}

To expand ILC populations for in vitro experiments, SCID mice were treated intranasally with $1 \mathrm{mg}$ of recombinant IL-33 (generated in-house; ${ }^{31}$ ), IL-12 (eBiosciences) or IL-18 $(\mathrm{R} \& \mathrm{D})$ at days 1,3 , and 5 and sacrificed at day 7. ILCs were enriched as described below for ex vivo analysis. For ILC2 population expansion, IL-33 was used ${ }^{49-51}$, and for ILC1 population expansion, IL-12 plus IL-18 plus IL-33 were used.

\section{Flow cytometry and cell sorting}

Mouse ILCs were stained with antibodies to CD3e (clone 145-2C11; 1:100; BD biosciences), CD49b (DX5; 1:200), TRAIL (N2B2; 1:100), NKp46 (29A1.4; 1:100), CD11a (M17/4; 1:100), IL-18Ra (P3TUNYA; 1:100, all eBiosciences), CD45 (30-F11; 1:400), CD25 (PC61; 1:400), CD90.2 (53-2.1; 1:400), CD44 (IM7; 1:400, all BioLegend) and ST2 (DJ8; 1:50, MDBiosciences). The lineage cocktail includes antibodies to TCR $\beta$ (H57-597), TCR $\gamma \delta$ (GL3), CD5 (53-7.3), F4/80 (BM8), CD11c (N418), Gr-1 (RB6-8C5), CD19 (1D3), FCeRIa (MAR-1) B220 (RA3-6B2), (all eBiosciences) and CD27 (LG3A10; BioLegend) (all used at 1:200). Intracellular antibodies included those to GATA-3 (TWAJ; 1:400), T-bet (4B10; 1:100), IL-13 (13A; 1:100), IL-5 (TRFK5; 1:100) and IFN- $\gamma$ (XMG1.2; 1:100, all eBiosciences). Live/dead fixable blue (1:100-1:300; Invitrogen) was used for all flow cytometry experiments. For intracellular cytokine staining of ILCs, cells were incubated with population expansion cytokines for time periods indicated in figures and legends, followed by $2-4 \mathrm{~h}$ of stimulation with PMA/Ionomycin and Brefeldin A before being 
surface stained, fixed and permeabilized (FoxP3 staining kit, eBiosciences) for intracellular staining.

For staining of human ILCs (Fig. 8) from patients with COPD, PBMCs from non-smoking healthy controls, smoking controls or stable patients with COPD were isolated from Heparin CP Tubes (BD biosciences) and depleted of T cells and B cells using CD3 and CD19 microbeads (Miltenyi) according to the manufacturer's protocol. All donors were drawn from the COPDgene pool ${ }^{52}$ and provided informed consent according to study guidelines. Cells were then stained with antibodies to CD3 (SK7; 2.5:125), CD19 (SJ25C1; 2.5:125, all BD Bioscience), IL-7Ra (RDR5; 3:125), CD161 (HP-3G10, 5:125, all eBiosciences), CRTH2 (BM16; 4:125) and CD56 (HCD56; 3:125) (BioLegend). The lineage cocktail includes antibodies against TCRa $\beta$ (IP26), TCR $\gamma \delta$ (B1), CD34 (561), CD14 (HCD14), CD16 (CB16), CD1a (HI149), CD303 (201A), CD123 (6H6), and FceRIa (AER-137; all eBiosciences) all used at a dilution of 2:125. Intracellular antibodies included those to GATA-3 (TWAJ; 1:400) and T-bet (4B10; 1:100, eBiosciences). Live/dead fixable blue (1:300; Invitrogen) was used for all flow cytometry experiments. All samples were run on an LSR II for flow cytometry or an Aria II for cell sorting (BD biosciences) and analyzed using FlowJo (Tree Star).

\section{ILC enrichment (mouse lung)}

Lungs were perfused with PBS, diced into $\sim 2-\mathrm{cm}$ pieces and incubated with Liberase TM $(42.4 \mu \mathrm{g} / \mathrm{ml})$ and DNAse I ( $10 \mathrm{U} / \mathrm{ml}$; both from Roche) for $45 \mathrm{~min}$ at $37^{\circ} \mathrm{C}$ before being mashed through a $70-\mu \mathrm{M}$ cell strainer and washed with complete RPMI. Remaining blood cells were lysed with ACK cell lysing buffer (Invitrogen) and single cell suspensions were incubated with biotinylated antibodies to CD3 $\varepsilon$ (clone 145-2C11), CD19 (1D3), B220 (RA3-6B2), CD5 (53-7.3), TCR $\beta$ (H57-597), TCR $\gamma \delta$ (GL3), CD11c (N418), F4/80 (BM8), Gr-1 (RB6-8C5), Ter119 (TER-119), CD49b (DX5; all eBioscience) and CD27 (LG3A10, BioLegend) all at $25 \mu \mathrm{g} / \mathrm{ml}$ per $2 \times 10^{8}$ cells. Cells were then incubated with anti-biotin microbeads (Miltenyi; 1:5 dilution at $10^{8}$ cells per $\mathrm{ml}$ ) and depleted following manufacturer's protocol. For each ILC enrichment, the depletion was repeated twice and yielded $>95 \%$ pure ILC populations (Supplementary Fig. 3a).

\section{Liver preparation}

Livers were perfused with PBS and diced into small pieces and then homogenized manually through a $70-\mu \mathrm{M}$ filter. After filtration, homogenized livers were mixed with Percoll Plus (GE, \#17-5445-02) and purified cells were extracted and red blood cells were lysed in ACK buffer. Cells were sorted using antibodies identified below (in 'Fluidigm analysis').

\section{Enrichment for B cells, T cells and NK cells (mouse)}

Lungs were treated as above and single-cell suspensions were incubated with biotinylated antibodies to CD19, CD3e, and CD49b (antibodies described above), followed by anti-biotin microbeads (Miltenyi) and enriched following the manufacturer's protocol. Positively selected B cells, T cells and NK cells were then prepared in PBS for further treatment. 


\section{ILC isolation for in vitro culture (human)}

Blood was collected from healthy volunteers recruited by the MedImmune Blood Donor program. All volunteers gave informed consent. PBMCs were isolated using CPT tubes according to the manufacturer's protocol (BD biosciences) and remaining red blood cells lysed using ACK lysis buffer. PBMCs from three to four healthy donors were pooled and depleted using an NK cell enrichment kit (StemCell technologies, Supplementary Fig. 7a). ILCs were then sorted to $99 \%$ purity as viable, $\mathrm{CD}^{4} 5^{+}$, non-T, non-B, $\mathrm{Lin}^{-}, \mathrm{IL}-7 \mathrm{Ra}^{+}$,

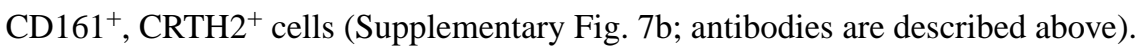

\section{Ex vivo stimulation of ILCs}

ILCs were isolated from murine lung or human blood as above and stimulated with IL-2, IL-7, IL-33, IL-12, IL-18, IL-15 or combinations thereof as stated in the figure. All cytokines were used at $50 \mathrm{ng} / \mathrm{ml}$. Cells were analyzed by flow cytometry and supernatants were assayed for cytokine production by MSD or ELISA (MesoScale Diagnostics).

\section{COPD samples}

Blood was collected from healthy control subjects, smoking control subjects and patients with COPD enrolled in the COPDGene cohort ${ }^{52}$. Patient demographics are given in Supplementary Tables 1 and 2. All work was approved by the Institutional Review Board at National Jewish Health. All patients provided written consent for the work performed.

\section{Fluidigm analysis}

ILC2 cells (defined as viable $\mathrm{CD} 45^{+} \mathrm{CD} 3 \varepsilon^{-} \mathrm{CD} 49 \mathrm{~b}^{-} \mathrm{Lin}^{-} \mathrm{CD} 90^{+} \mathrm{ST} 2^{+} \mathrm{IL}-18 \mathrm{Ra}{ }^{-}$) were sorted from naive lungs. ILC2 cells (viable CD $45^{+} \mathrm{CD} 3 \varepsilon^{-} \mathrm{CD} 49 \mathrm{~b}^{-} \mathrm{Lin}^{-} \mathrm{CD} 90^{+} \mathrm{ST} 2^{+} \mathrm{IL}-18 \mathrm{R}$ $\mathrm{a}^{-}$) and ILC1 cells (viable CD45+CD3 $\varepsilon{ }^{-} \mathrm{C}$ D $49 b^{-} \mathrm{Lin}^{-} \mathrm{CD} 90^{+} \mathrm{ST} 2-\mathrm{IL}-18 \mathrm{Ra}^{+}$) were sorted from lungs of mice treated with IL-12 plus IL-18 plus IL-33 as described above; for controls, NK cells (viable CD45 ${ }^{+} \mathrm{CD} 49 \mathrm{~b}^{+} \mathrm{CD} 3 \varepsilon^{-}$) were sorted from naive lung and ILC1s were sorted from naive liver (viable CD $45^{+} \mathrm{CD} 3 \varepsilon{ }^{-} \mathrm{CD} 19^{-} \mathrm{NKp} 46^{-} \mathrm{CD} 11 \mathrm{a}^{+} \mathrm{TRAIL}^{+}$). All antibody clones described above. RNA was isolated from cells and qPCR was performed using the Fluidigm Biomark Dynamic array loaded with probes for transcripts of interest (Fluidigm Corp., South San Francisco, Calif, USA).

\section{Adoptive transfer of ILCs}

For ILC2 transfers (Figs. 4-6), mice were treated intranasally on days 1, 3 and 5 with $2.5 \mu \mathrm{g}$ of recombinant IL-33 for ILC2 population expansion, or IL-12 plus IL-18 plus IL-33 for ILC1 population expansion. On day 7, viable $\mathrm{CD} 45^{+} \mathrm{CD} 3 \varepsilon{ }^{-} \mathrm{CD} 49 \mathrm{~b}^{-} \mathrm{Lin}^{-} \mathrm{CD} 90^{+} \mathrm{CD} 44^{+} \mathrm{CD} 25{ }^{+} \mathrm{ST} 2{ }^{+} \mathrm{IL}-18 \mathrm{Ra}{ }^{-}$cells were purified and 1 $\times 10^{5}$ to $1.5 \times 10^{5}$ were transferred via tail vein injections (all antibody clones used are described above). $2.5 \times 10^{6}$ to $3 \times 10^{6}$ lung-derived T cells, B cells and NK cells were transferred in addition to ILC2 cells. Twelve hours later, recipient mice were infected with influenza A virus, analysis was done on days 6,7 and day 10 post-infection as indicated in the figure legends. Cytokines in the BAL were measured by MSD (MesoScale Diagnostics) at day 2 after infection. 


\section{Immunohistochemical identification of $\mathrm{GFP}^{+}$cells}

$3-\mu \mathrm{m}$ thick paraffin-embedded lung sections were subjected to heat-induced epitope retrieval (HIER) in a pretreatment module (PT-link; Dako Cytomation) with citrate buffer $(\mathrm{pH}=6)$ before immunohistochemical staining. The staining was performed at room temperature in an automated IHC robot (AutostainerPlus; Dako). Briefly, sections were sequentially blocked with endogenous enzyme block (EnVision FLEX Peroxidase-Blocking Reagent; Dako) and serum free protein block (Dako) before incubation with a primary chicken antiGFP antibody (1:3,000; Abcam; \#ab13970). Next, sections were incubated with a Polymer/ HRP-linked secondary antibody (Flex kit, Dako) per the manufacturer's instructions, followed by incubation with 3,3' -diaminobenzidine (DAB) substrate-chromogen solution for $10 \mathrm{~min}$ and counterstaining with Mayer's hematoxylin (blue nuclei). Finally, sections were dehydrated through ethanol series, cleared in xylene and mounted with Pertex (HistoLab).

\section{Double immunohistochemical staining of GFP+ cells and influenza A virus}

Immunoreactivity for GFP and influenza A virus was co-visualized in mouse lungs. Briefly, HIER-treated sections were incubated with chicken anti-GFP antibodies (antibody described above). The anti-GFP antibody was detected by goat anti-chicken antibodies (1:200; Abcam; \#ab6877) conjugated to HR-Peroxidase (HRP) and a brown-colored immunoreaction product was produced using the peroxidase substrate DAB as a chromogen. The sections were then treated with denaturing solution (Biocare Medical), and incubated with goat antiInfluenza A virus antibodies (1:1,000; Abcam; \#ab20841), followed by incubation with a HRP-conjugated rabbit anti-goat antibody (1:200; Dako; \#P044901-2). Finally, a greencolored influenza virus immunoreaction product was produced using the peroxidase substrate Vina Green as chromogen (Biocare Medical). Hematoxylin was used as background staining (blue nuclei) and the tissue sections were air dried, cleared in xylene and mounted with Pertex (HistoLab).

\section{Triple immunohistochemical staining of GFP, GATA-3 and influenza A virus}

HIER-treated tissue sections were incubated with goat anti-influenza A virus antibody, followed by a HRP-conjugated rabbit anti-goat antibody (described above) and development of the brown DAB chromogen at the site of influenza virus immunoreactivity. Next, sections were treated with denaturing blocking solution and incubated with chicken anti-GFP (described above) and rabbit anti-GATA3 primary antibodies (1:100; Abcam; \#ab106625). This was followed by incubation with goat anti-chicken (1:500; \#A-11039) and goat antirabbit (1:1,000; \#A-27039) secondary antibodies conjugated to Alexa Fluor (AF) 488 or AF555, respectively (Life Technologies). Finally, the tissue was treated with the DNAbinding fluorochrome Hoechst (blue nuclei), and mounted with PBS/glycerol. The immunoreactivity for all 3 markers was captured and digitized by a combined bright field and epifluoroscence digital slide scanner unit (Olympus VS120).

\section{In situ hybridization (ISH)}

$I 112$ and $I 118$ mRNA was visualized using the RNAscope 2.0 FFPE assay kit according to the manufacturer's instructions (Advanced Cell Diagnostics). Briefly, tissue sections were 
deparaffinized, incubated with endogenous enzyme block, boiled in pretreatment buffer and treated with protease, followed by target probe hybridization using Mm-IL12b (319551, ACD) and Mm-IL18 (416731, ACD) probes. Probes against the housekeeping gene PPIB or the bacterial gene DapB served as positive and negative controls respectively. The target RNA was then amplified and detected with DAB chromogen. Finally, the tissue sections were dehydrated and mounted using Pertex. For combined ISH tissue sections previously stained for $I 112$ and $I 118$ mRNA through in situ hybridization were subjected to subsequent IHC through incubation with a chicken anti-GFP antibody (described above) followed by detection by a goat anti chicken-HRP (described above) and Vina Green chromogen development.

\section{Quadruple histochemistry and quantification of GATA-3 intensity in GFP+ cells}

Combined immunofluorescence and chromogenic IHC was used to examine and quantify GATA-3 associated ILC expression. Sections were subjected to DNA/nuclear staining (DAPI, Alexa 355) combined with triple immunohistochemical staining for GFP, GATA-3 (EPR16651; immunofluoroscence with AF488 and AF555, respectively), as well as influenza virus (ab20841; bright field visualization with DAB chromogen). All staining channels were digitized by a combined fluorescence and bright field Olympus VS-120 virtual slide microscope to generate one high-resolution image of the entire section for each marker. The intensity of the GATA-3 immunostaining in individual $\mathrm{GFP}^{+}$cells was measured using an automated region-of-interest (ROI) based method (software ImageJ $1.47 \mathrm{v})$. Briefly, starting with the AF488 image, intensity threshold was adjusted, locked, and used to create ROIs corresponding to $\mathrm{GFP}^{+}$cells. The multiple GFP ROIs were then pasted into the corresponding Alexa555 image and used to measure the intensity of GATA-3 (i.e. Alexa555 mean intensity) in each $\mathrm{GFP}^{+}$ROI. Similarly, the GFP ROIs were pasted into the AF355 image and the intensity of DAPI in the same $\mathrm{GFP}^{+}$ROIs was calculated in order to adjust the GATA-3 intensity for nuclear content. The scanned bright field image of influenza staining was used to visualize the spatial relationship between $\mathrm{GFP}^{+}$cells, GATA-3 intensity and regional ongoing infection.

\section{Quantification of influenza A virus in GFP+ cells}

Sections were subjected to immunohistochemical staining for GFP and influenza A virus (bright field visualization with DAB and Vina Green, respectively) and digitalized by Olympus VS-120 virtual slide microscope. Total immunoreactivity of influenza A virus and the total tissue area were calculated using computerized image analysis (Visiomorph-DP, Denmark). Influenza A virus positivity was calculated as the percent of the total tissue area with influenza A virus immunoreactivity.

\section{Statistical analysis}

For COPD samples, the one-way mixed-effect ANOVA model with heterogeneous within group variance was applied for comparisons of continuous measurements and the linear contrast was used to test linear trend between measurements and levels of average exacerbations per year. Fisher's exact test was used for comparison of categorical measurements and Wilcoxon test was used to compare average exacerbations per year in the demographic tables. The Pearson correlation coefficient was used to evaluate correlation 
between the two continuous measurements. SAS 9.3 was used for the statistical analysis and $P<0.05$ was considered significant.

For all other samples, data were analyzed and expressed as stated in the figure legends using GraphPad Prism. Error bars represent s.e.m. For analysis of experiments containing two groups, the Mann-Whitney-Wilcoxon test was used to determine significance. For experiments containing more than two groups, a one-way ANOVA with Tukey post-test was performed. For correlations, regression analysis was performed. For all statistical analysis, $P$ $<0.01$ was considered significant.

\section{Supplementary Material}

Refer to Web version on PubMed Central for supplementary material.

\section{Acknowledgments}

We thank the MedImmune Flow Cytometry core for all cell sorting; the MedImmune histology core for embedding tissues; the LAR staff for maintaining the experimental mice; M. Stämpfli for expertise and guidance in establishing a smoking system at MedImmune; A. Gonzales for running and maintaining the system for in-house exposure to cigarette smoke; the C. Lopez laboratory (University of Pennsylvania, School of Veterinary Medicine) for influenza virus strain PR8; M. Snaith for help with generating the ST2-GFP reporter mouse; M.E.P. Roberts for facilitating the collaboration with National Jewish Health; C. Schnell, T. Thorn and the rest of the team at NJH for commitment and contributions to this collaborative effort; and J. Jönsson and K. Jansner for histological work and image processing and analysis.

\section{References}

1. Artis D, Spits H. The biology of innate lymphoid cells. Nature. 2015; 517:293-301. [PubMed: 25592534]

2. McKenzie AN, Spits H, Eberl G. Innate lymphoid cells in inflammation and immunity. Immunity. 2014; 41:366-374. [PubMed: 25238094]

3. Spits H, et al. Innate lymphoid cells--a proposal for uniform nomenclature. Nat Rev Immunol. 2013; 13:145-149. [PubMed: 23348417]

4. Bernink JH, et al. Human type 1 innate lymphoid cells accumulate in inflamed mucosal tissues. Nat Immunol. 2013; 14:221-229. [PubMed: 23334791]

5. Daussy C, et al. T-bet and Eomes instruct the development of two distinct natural killer cell lineages in the liver and in the bone marrow. J Exp Med. 2014; 211:563-577. [PubMed: 24516120]

6. Fuchs A, et al. Intraepithelial type 1 innate lymphoid cells are a unique subset of IL-12- and IL-15responsive IFN- $\gamma$-producing cells. Immunity. 2013; 38:769-781. [PubMed: 23453631]

7. Klose CS, et al. Differentiation of type 1 ILCs from a common progenitor to all helper-like innate lymphoid cell lineages. Cell. 2014; 157:340-356. [PubMed: 24725403]

8. Furusawa J, et al. Critical role of p38 and GATA3 in natural helper cell function. J Immunol. 2013; 191:1818-1826. [PubMed: 23851685]

9. Liang HE, et al. Divergent expression patterns of IL-4 and IL-13 define unique functions in allergic immunity. Nat Immunol. 2012; 13:58-66.

10. Hoyler T, Connor CA, Kiss EA, Diefenbach A. T-bet and Gata3 in controlling type 1 and type 2 immunity mediated by innate lymphoid cells. Curr Opin Immunol. 2013; 25:139-147. [PubMed: 23490163]

11. Mjösberg J, et al. The transcription factor GATA3 is essential for the function of human type 2 innate lymphoid cells. Immunity. 2012; 37:649-659. [PubMed: 23063330]

12. Cella M, et al. A human natural killer cell subset provides an innate source of IL-22 for mucosal immunity. Nature. 2009; 457:722-725. [PubMed: 18978771] 
13. Luci C, et al. Influence of the transcription factor ROR $\gamma \mathrm{t}$ on the development of $\mathrm{NKp} 46^{+}$cell populations in gut and skin. Nat Immunol. 2009; 10:75-82. [PubMed: 19029904]

14. Satoh-Takayama N, et al. Microbial flora drives interleukin 22 production in intestinal NKp46 ${ }^{+}$ cells that provide innate mucosal immune defense. Immunity. 2008; 29:958-970. [PubMed: 19084435]

15. Sanos SL, Vonarbourg C, Mortha A, Diefenbach A. Control of epithelial cell function by interleukin-22-producing ROR $\gamma \mathrm{t}^{+}$innate lymphoid cells. Immunology. 2011; 132:453-465. [PubMed: 21391996]

16. Diefenbach A, Colonna M, Koyasu S. Development, differentiation, and diversity of innate lymphoid cells. Immunity. 2014; 41:354-365. [PubMed: 25238093]

17. Cella M, Otero K, Colonna M. Expansion of human NK-22 cells with IL-7, IL-2, and IL-1 $\beta$ reveals intrinsic functional plasticity. Proc Natl Acad Sci USA. 2010; 107:10961-10966. [PubMed: 20534450]

18. Hughes T, et al. Stage 3 immature human natural killer cells found in secondary lymphoid tissue constitutively and selectively express the $\mathrm{T}_{\mathrm{H}} 17$ cytokine interleukin-22. Blood. 2009; 113:40084010. [PubMed: 19244159]

19. Hughes $T$, et al. Interleukin- $1 \beta$ selectively expands and sustains interleukin- $22^{+}$immature human natural killer cells in secondary lymphoid tissue. Immunity. 2010; 32:803-814. [PubMed: 20620944]

20. Klose CSN, et al. A T-bet gradient controls the fate and function of CCR6 ${ }^{-} \mathrm{ROR}_{\mathrm{\gamma t}}{ }^{+}$innate lymphoid cells. Nature. 2013; 494:261-265. [PubMed: 23334414]

21. Vonarbourg C, et al. Regulated expression of nuclear receptor ROR $\gamma \mathrm{t}$ confers distinct functional fates to NK cell receptor-expressing ROR $\gamma \mathrm{t}^{+}$innate lymphocytes. Immunity. 2010; 33:736-751. [PubMed: 21093318]

22. Sciumé G, et al. Distinct requirements for T-bet in gut innate lymphoid cells. J Exp Med. 2012; 209:2331-2338. [PubMed: 23209316]

23. Bernink JH, et al. Interleukin-12 and -23 control plasticity of $\mathrm{CD} 127^{+}$group 1 and group 3 innate lymphoid cells in the intestinal lamina propria. Immunity. 2015; 43:146-160. [PubMed: 26187413]

24. Crellin NK, et al. Regulation of cytokine secretion in human CD $127^{+}$LTi-like innate lymphoid cells by Toll-like receptor 2. Immunity. 2010; 33:752-764. [PubMed: 21055975]

25. Sethi S, Murphy TF. Infection in the pathogenesis and course of chronic obstructive pulmonary disease. N Engl J Med. 2008; 359:2355-2365. [PubMed: 19038881]

26. MacDonald M, Korman T, King P, Hamza K, Bardin P. Exacerbation phenotyping in chronic obstructive pulmonary disease. Respirology. 2013; 18:1280-1281. [PubMed: 24164687]

27. Mallia P, et al. Experimental rhinovirus infection as a human model of chronic obstructive pulmonary disease exacerbation. Am J Respir Crit Care Med. 2011; 183:734-742. [PubMed: 20889904]

28. Mohan A, et al. Prevalence of viral infection detected by PCR and RT-PCR in patients with acute exacerbation of COPD: a systematic review. Respirology. 2010; 15:536-542. [PubMed: 20415983]

29. Botelho FM, et al. IL-1a/IL-1R1 expression in chronic obstructive pulmonary disease and mechanistic relevance to smoke-induced neutrophilia in mice. PLoS One. 2011; 6:e28457. [PubMed: 22163019]

30. Kang MJ, et al. IL-18 is induced and IL-18 receptor a plays a critical role in the pathogenesis of cigarette smoke-induced pulmonary emphysema and inflammation. J Immunol. 2007; 178:19481959. [PubMed: 17237446]

31. Kearley J, et al. Cigarette smoke silences innate lymphoid cell function and facilitates an exacerbated type I interleukin-33-dependent response to infection. Immunity. 2015; 42:566-579. [PubMed: 25786179]

32. Iwasaki A, Pillai PS. Innate immunity to influenza virus infection. Nat Rev Immunol. 2014; 14:315-328. [PubMed: 24762827]

33. Lazarevic V, Glimcher LH, Lord GM. T-bet: a bridge between innate and adaptive immunity. Nat Rev Immunol. 2013; 13:777-789. [PubMed: 24113868] 
34. Yagi R, et al. The transcription factor GATA3 is critical for the development of all IL-7Raexpressing innate lymphoid cells. Immunity. 2014; 40:378-388. [PubMed: 24631153]

35. Monticelli LA, et al. Innate lymphoid cells promote lung-tissue homeostasis after infection with influenza virus. Nat Immunol. 2011; 12:1045-1054. [PubMed: 21946417]

36. Regan EA, et al. Genetic epidemiology of COPD (COPDGene) study design. COPD. 2010; 7:3243. [PubMed: 20214461]

37. Ohne, Y., et al. IL-1 is a critical regulator of group 2 innate lymphoid cell function and plasticity. Nat Immunol. Apr 25. 2016 http://dx.doi.org/10.1038/ni.3447

38. Serafini N, et al. Gata3 drives development of ROR $\gamma \mathrm{t}^{+}$group 3 innate lymphoid cells. J Exp Med. 2014; 211:199-208. [PubMed: 24419270]

39. Zhong C, et al. Group 3 innate lymphoid cells continuously require the transcription factor GATA-3 after commitment. Nat Immunol. 2016; 17:169-178. [PubMed: 26595886]

40. Califano D, et al. Transcription factor Bcl11b controls identity and function of mature type 2 innate lymphoid cells. Immunity. 2015; 43:354-368. [PubMed: 26231117]

41. Bal, SM., et al. Interleukin-1 $\beta,-4$ and -12 control ILC2 fate in human airway inflammation. Nat Immunol. Apr 25. 2016 http://dx.doi.org/10.1038/ni.3444

42. Vignali DAA, Kuchroo VK. IL-12 family cytokines: immunological playmakers. Nat Immunol. 2012; 13:722-728. [PubMed: 22814351]

43. Molofsky AB, et al. Interleukin-33 and Interferon- $\gamma$ counter-regulate group 2 innate lymphoid cell activation during immune perturbation. Immunity. 2015; 43:161-174. [PubMed: 26092469]

44. Duerr CU, et al. Type I interferon restricts type 2 immunopathology through the regulation of group 2 innate lymphoid cells. Nat Immunol. 2016; 17:65-75. [PubMed: 26595887]

45. Moro K, et al. Interferon and IL-27 antagonize the function of group 2 innate lymphoid cells and type 2 innate immune responses. Nat Immunol. 2016; 17:76-86. [PubMed: 26595888]

46. Sojka DK, et al. Tissue-resident natural killer (NK) cells are cell lineages distinct from thymic and conventional splenic NK cells. eLife. 2014; 3:e01659. [PubMed: 24714492]

47. Bauer CMT, Morissette MC, Stämpfli MR. The influence of cigarette smoking on viral infections: translating bench science to impact COPD pathogenesis and acute exacerbations of COPD clinically. Chest. 2013; 143:196-206. [PubMed: 23276842]

48. Byers DE, et al. Long-term IL-33-producing epithelial progenitor cells in chronic obstructive lung disease. J Clin Invest. 2013; 123:3967-3982. [PubMed: 23945235]

49. Neill DR, et al. Nuocytes represent a new innate effector leukocyte that mediates type- 2 immunity. Nature. 2010; 464:1367-1370. [PubMed: 20200518]

50. Moro $\mathrm{K}$, et al. Innate production of $\mathrm{T}_{\mathrm{H}} 2$ cytokines by adipose tissue-associated c-Kit ${ }^{+} \mathrm{Sca}-1^{+}$ lymphoid cells. Nature. 2010; 463:540-544. [PubMed: 20023630]

51. Price AE, et al. Systemically dispersed innate IL-13-expressing cells in type 2 immunity. Proc Natl Acad Sci USA. 2010; 107:11489-11494. [PubMed: 20534524]

52. Roberts MEP, et al. CD4 ${ }^{+}$T-Cell profiles and peripheral blood ex-vivo responses to T-cell directed stimulation delineate COPD phenotypes. J COPD Found. 2016; 2:268-280. 

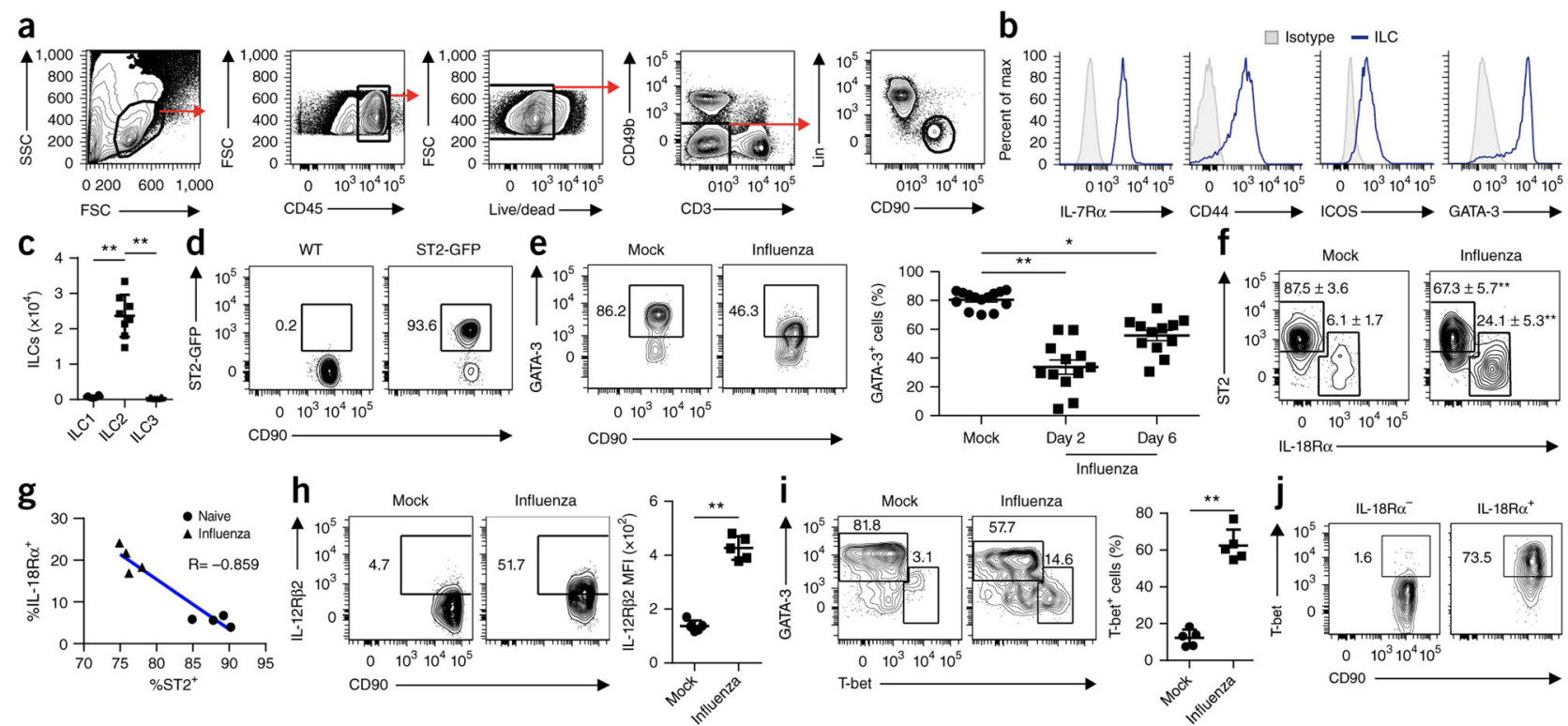

j
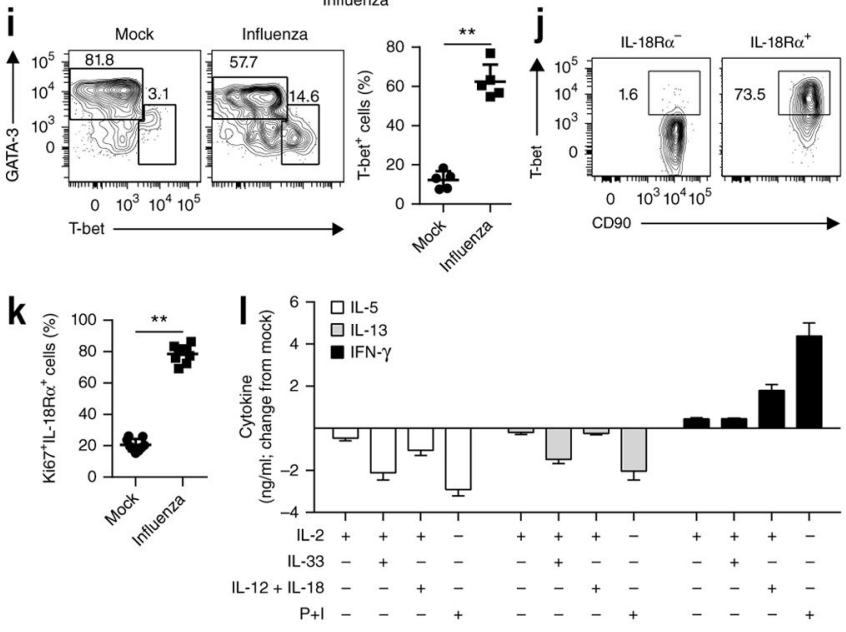

Figure 1.

Infection with influenza virus triggers downregulation of GATA-3 expression in lungresident ILCs. (a) Flow cytometry gating lung-resident ILCs as $\mathrm{CD}^{4} 5^{+}$, viable, $\mathrm{CD} 4 \mathrm{~b}^{-} \mathrm{CD}^{-}$and $\mathrm{Lin}^{-} \mathrm{CD} 90^{+}$. SSC, side scatter; FSC, forward scatter. (b) Expression of ILC-associated markers on $\mathrm{Lin}^{-} \mathrm{CD} 90^{+}$ILCs. Isotype, isotype-matched control antibody. (c) Quantification of ILC1, ILC2 and ILC3 cells in naive mice on the basis of transcriptionfactor expression. (d) Flow cytometry of ILCs from ST2-GFP reporter mice and their wildtype littermates (WT). Numbers adjacent to outlined areas indicate percent ST2-GFP ${ }^{+}$ILCs. (e) Flow cytometry of ILCs from mock-treated mice (Mock) and influenza-virus-infected mice (Influenza) at day 7 after infection (left), and frequency of GATA- $3^{+}$cells in such mice after mock infection or at day 2 or day 6 after viral infection (right). Numbers adjacent to outlined areas (left) indicate percent GATA-3 ILCs. (f) Flow cytometry of cells from mice as in e. Numbers adjacent to outlined areas indicate percent (mean \pm s.e.m.) ST2 $2^{+} \mathrm{IL}_{-} 18 \mathrm{Ra}{ }^{-}$ cells (left) or ST2-IL-18Ra ${ }^{+}$cells (right). (g) Correlation of the expression of ST2 and IL-18Ra on ILCs from naive and infected mice. $R=-0.859$. (h) Flow cytometry analyzing IL-12R $\beta 2$ expression by ILCs from mice as in $\mathbf{e}$ (left), and mean fluorescence intensity (MFI) of IL-12R $\beta 2$ on those cells (right). Numbers adjacent to outlined areas (left) indicate percent IL-12R $\beta 2^{+}$ILCs. (i) Flow cytometry analyzing the expression of GATA-3 and T-bet by ILCs from mice as in $\mathbf{e}$ (left), and frequency of T-bet ${ }^{+}$cells (right). Numbers adjacent to outlined areas (left) indicate percent GATA- ${ }^{+}$T-bet $^{-}$cells (top left) or GATA- $3^{-}{ }^{-}$-bet ${ }^{+}$cells (bottom right). (j) Flow cytometry of IL-18Ra ${ }^{-}$and IL-18Ra ${ }^{+}$ILCs (above plots) from 
mice at day 7 after infection with influenza virus. Numbers adjacent to outlined areas indicate percent T-bet ${ }^{+}$ILCs. (k) Frequency of Ki $67^{+}$cells expressing IL-18Ra in mice at day 7 after infection as in e. (l) Cytokine levels in supernatants from cultures of enriched ILCs obtained from mice at day 7 after infection with influenza virus and stimulated with various combinations (below plot) of cytokines or with the phorbol ester PMA and ionomycin $(\mathrm{P}+\mathrm{I})$, presented relative to that in naive mice. Each symbol $(\mathbf{c}, \mathbf{e}, \mathbf{h}, \mathbf{i}, \mathbf{k})$ represents an individual mouse; small horizontal lines indicate the mean ( \pm s.e.m.). ${ }^{*} P<0.01$ and $* * P$ $<0.001$ (Mann-Whitney Wilcoxon test). Data are representative of five independent experiments with eight $(\mathbf{a}-\mathbf{c}, \mathbf{f}, \mathbf{g})$, twelve (e) or five (h,i) mice per group (a-c,e-i; mean in $\mathbf{e , h}, \mathbf{i}(\mathrm{left}))$ or two independent experiments with nine mice per group (k,l; mean + s.e.m. in l) or are pooled from two independent experiments with three (d) or five (j) mice per group (d,j; mean). 


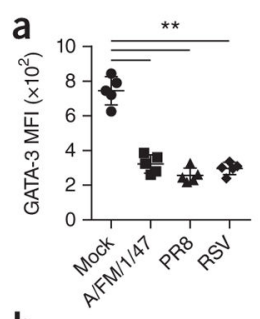

h

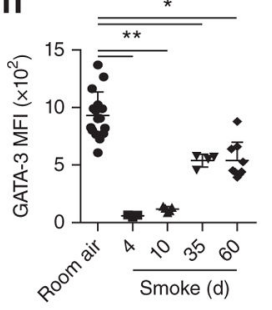

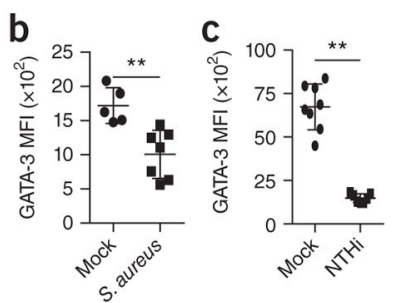

i

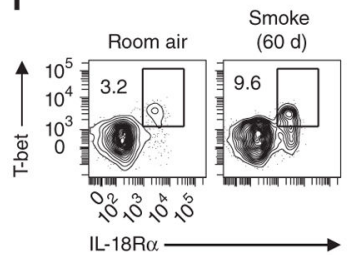

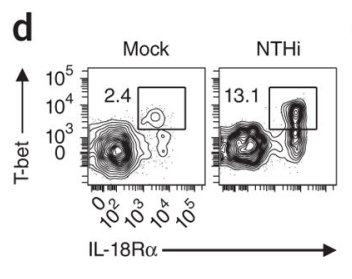
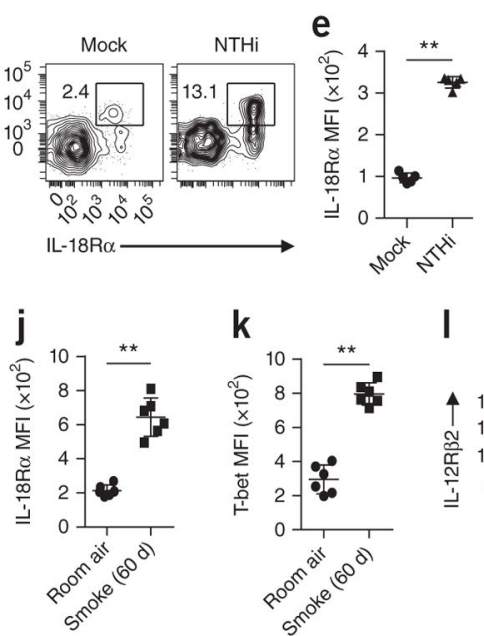

K

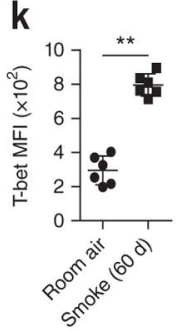

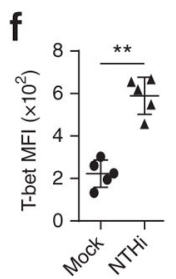

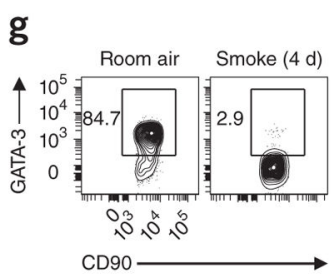

I

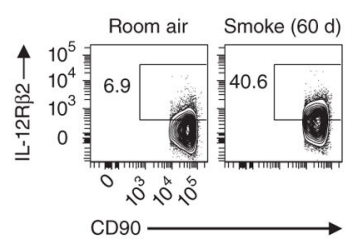

m

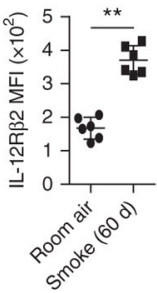

Figure 2.

Various COPD-associated triggers alter ILC populations. (a-c) MFI of GATA-3 in lungresident ILCs from mice mock infected or infected with influenza A virus strain A/FM/1/47 or PR8 or respiratory syncytial virus (RSV) (a), S. aureus (b) or nontypeable $H$. influenzae (NTHi) (c). (d) Flow cytometry of lung-resident ILCs from mice at day 5 after mock infection or infection with NTHI. Numbers adjacent to outlined areas indicate percent Tbet $^{+}$IL-18Ra ${ }^{+}$cells. (e,f) MFI of IL-18Ra (e) and T-bet (f) on cells as in d. (g) Flow cytometry of lung-resident ILCs from mice exposed for $4 \mathrm{~d}$ to room air or cigarette smoke (above plots). Numbers adjacent to outlined areas indicate percent GATA- $3^{+}$ILCs. (h) MFI of GATA-3 in lung-resident ILCs from mice exposed for $4 \mathrm{~d}$ to room air or for $4,10,35$ or $60 \mathrm{~d}$ to cigarette smoke. (i) Flow cytometry of lung-resident ILCs from mice exposed for 8 weeks $(60 \mathrm{~d})$ to room air or cigarette smoke. Numbers adjacent to outlined areas indicate percent T-bet ${ }^{+} \mathrm{IL}-18 \mathrm{Ra}^{+}$cells. $(\mathbf{j}, \mathbf{k}) \mathrm{MFI}$ of IL-18Ra $(\mathbf{j})$ and T-bet $(\mathbf{k})$ on cells as in $\mathbf{i}$. (l) Flow cytometry of lung-resident ILCs from mice exposed for $60 \mathrm{~d}$ to room air or cigarette smoke. Numbers adjacent to outlined areas indicate percent IL-12R $\beta 2^{+}$ILCs. (m) MFI of IL-12Rß2 on cells as in l. Each symbol $(\mathbf{a}-\mathbf{c}, \mathbf{e}, \mathbf{f}, \mathbf{h}, \mathbf{j}, \mathbf{k}, \mathbf{m})$ represents an individual mouse; small horizontal lines indicate the mean ( \pm s.e.m.). $* P<0.01$ and $* * P<0.001$ (MannWhitney Wilcoxon test). Data are representative of two independent experiments with five mice per group (a,b,e,f), three independent experiments with seven (c), fifteen (g, room air), three (g, smoke, $4 \mathrm{~d})$, five (g, smoke, $10 \mathrm{~d})$, four (g, smoke, $35 \mathrm{~d})$, nine (g, smoke, $60 \mathrm{~d})$, or six (i-m) mice per group $(\mathbf{c}, \mathbf{g}, \mathbf{i}-\mathbf{m}$; mean in $\mathbf{g , i}, \mathbf{l})$, four experiments (d; mean) or two experiments $(\mathbf{h})$. 
a

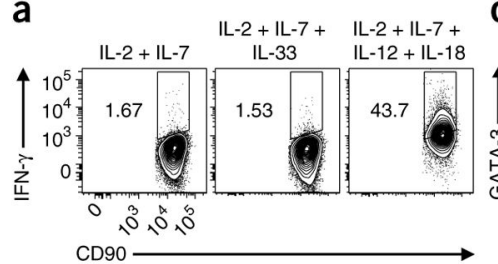

C

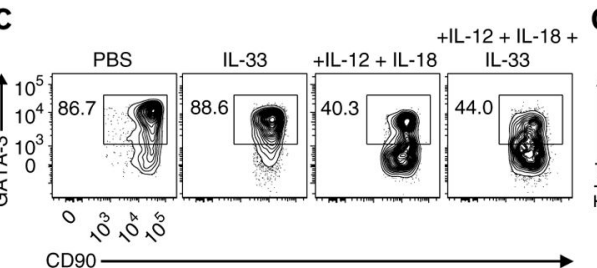

d
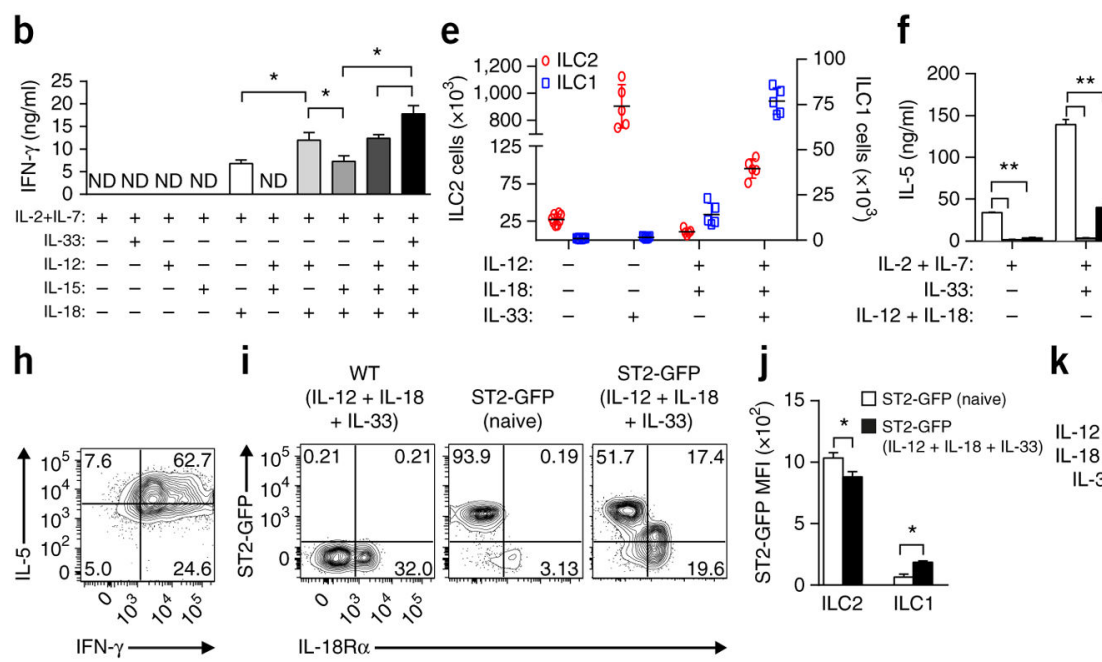

$+++++$

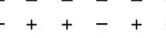

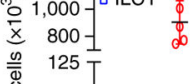

$\begin{array}{cc}\frac{\omega}{\bar{\Phi}} & 125 \\ \text { ठ } & 75 \\ \text { ปे } & \end{array}$
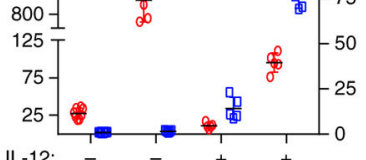

-18: -

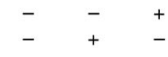

i $\quad$ WT

(IL-12 + IL-18 ST2-GFP $\quad$ (IL-12-GFP $\begin{array}{lll}+ \text { IL-33) } & \text { (naive) } \quad+\text { IL-33) }\end{array}$
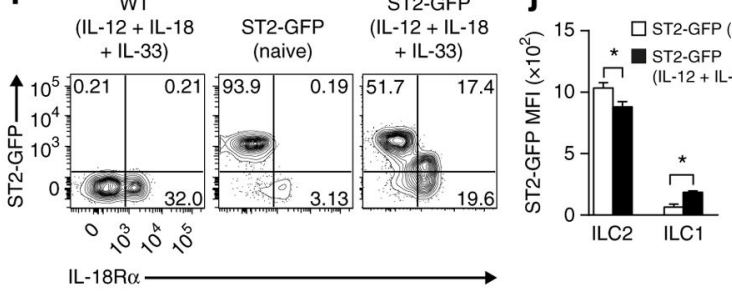
-

k$$
\text { k }
$$

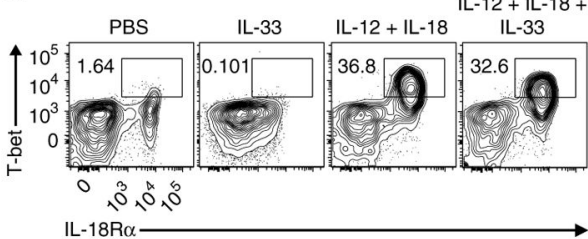

g 口иL-33

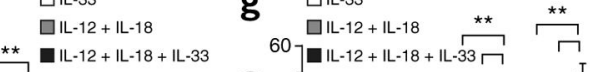
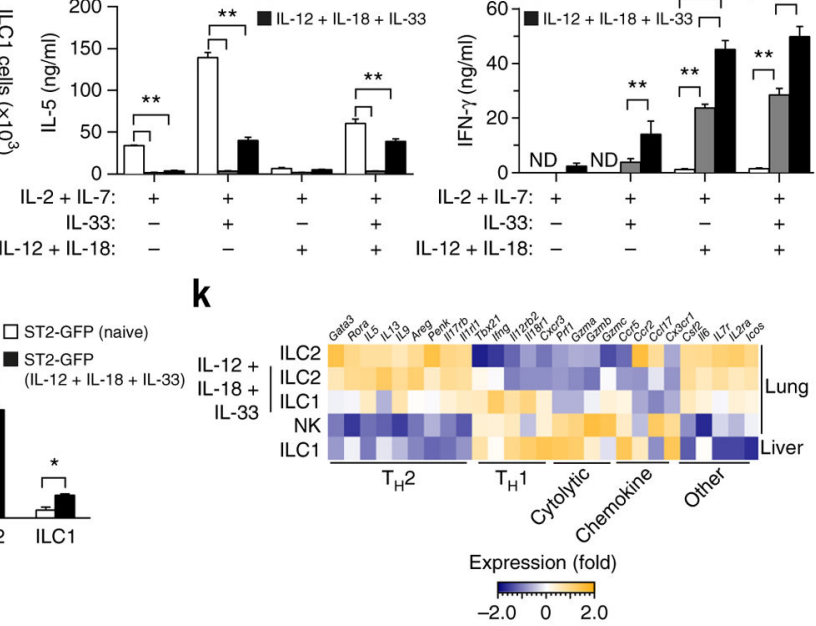

Figure 3.

ILC1 cells are derived from the local ST2 ${ }^{+}$ILC2 pool. (a) Flow cytometry of mouse lung ILC2 cells cultured for $96 \mathrm{~h}$ with various combinations of cytokines (above plots). Numbers adjacent to outlined areas indicate percent IFN- $\gamma^{+}$ILCs. (b) Concentration of IFN- $\gamma$ in ILCs cultured with various combinations of cytokines (below plot). (c,d) Flow cytometry of ILCs from SCID mice given intranasal PBS, IL-33, IL-12 plus IL-18, or IL-12 plus IL-18 plus IL-33 (above plots). Numbers adjacent to outlined areas indicate percent GATA- $3^{+}$ILCs (c) or T-bet ${ }^{+}$IL-18Ra ${ }^{+}$cells (d). (e) Quantification of lung-resident ILC2 or ILC1 cells in mice treated with various combinations of IL-12, IL-18 and IL-33 (below plot). Each symbol represents an individual mouse; small horizontal lines indicate the mean ( \pm s.e.m.). (f,g) Concentration of IL-5 (f) or IFN- $\gamma(\mathbf{g})$ in supernatants of ILCs enriched from the lungs of mice treated with various combinations of IL-12, IL-18 and IL-33 (key) and stimulated in vitro for $24 \mathrm{~h}$ with IL-1 plus IL-7 and various combinations of IL-33, IL-12 and IL-18 (below plots). (h) Flow cytometry analyzing the expression of IL-5 and IFN- $\gamma$ by ILCs enriched from the lungs of mice treated with IL-12 plus IL-18 plus IL-33. Numbers in quadrants indicate percent cells in each throughout. (i) Flow cytometry analyzing the expression of ST2-GFP and IL-18Ra on lung-resident ILCs from naive ST2-GFP reporter mice or ST2-GFP reporter mice treated with IL-12 plus IL-18 plus IL-33 and their wild-type littermates (above plots). (j) MFI of ST2-GFP on ILC2 or ILC1 cells in ST2-GFP reporter mice as in i. (k) Quantitative PCR analysis of selected genes (above), categorized as $\mathrm{T}_{\mathrm{H}} 2$ or $\mathrm{T}_{\mathrm{H}} 1$ signature genes, or genes encoding products involved in the cytolytic pathway or chemokines, or other genes (below plot), in cells (left margin) sorted from lungs of naive mice (ILC2 and NK cells) or mice treated with IL-12 plus IL-18 plus IL-33 (active ILC2 cells $\left(\mathrm{ST}^{+}\right)$and active ILC1 cells (IL-18Ra $\left.{ }^{+}\right)$) or the liver of naive mice (ILC1 cells). ND, 
not detectable. $* P<0.01$ and $* * P<0.001$ (Mann-Whitney Wilcoxon test). Data are representative of three experiments $(\mathbf{a}-\mathbf{d}, \mathbf{f}-\mathbf{h}$; mean $(\mathbf{a}, \mathbf{c}, \mathbf{d})$ or mean + s.e.m. $(\mathbf{b}, \mathbf{f}, \mathbf{g})$ of four (a-d) or three (f,g) technical replicates), two independent experiments with nine mice per group (PBS) or five mice per group (all other conditions) (e) or two experiments (k) or are pooled from two independent experiments with three mice per group (naive) or four mice per group (cytokine treatment) $(\mathbf{i}, \mathbf{j})$. 

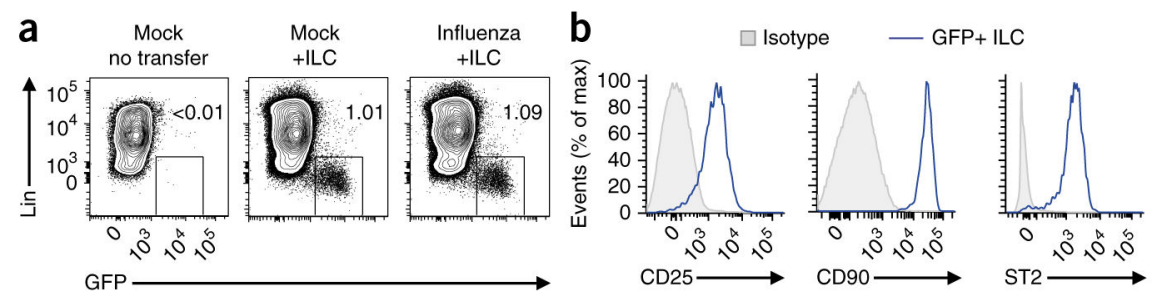

C
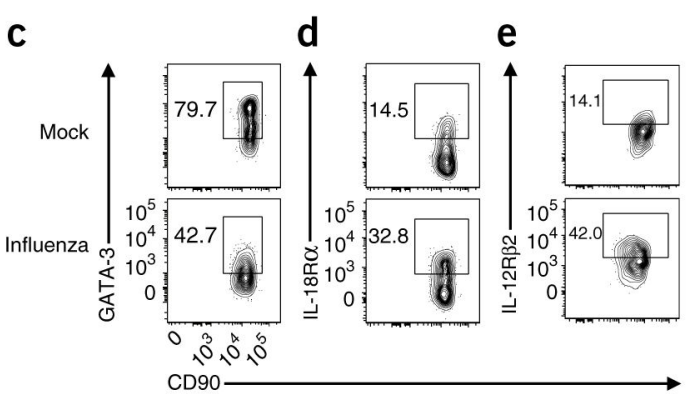

f $\quad \mathrm{g} \quad \mathrm{h}$

h

i
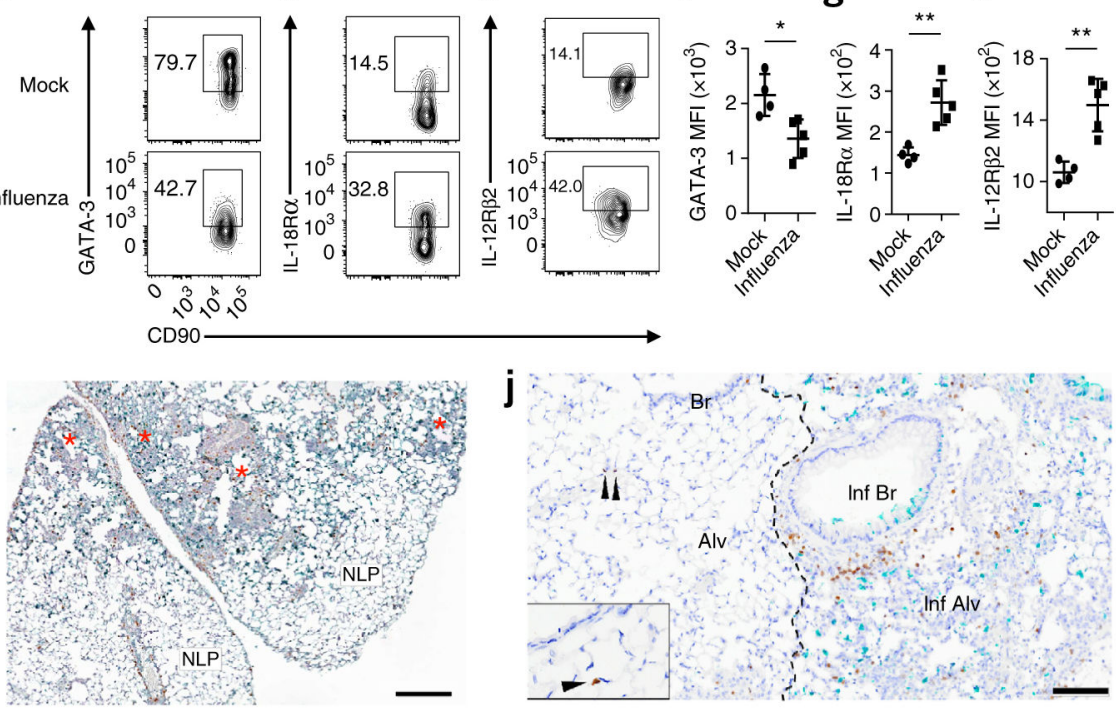

Figure 4.

Direct conversion of ILC2 cells into ILC1 cells during viral challenge. (a) Flow cytometry of cells from $\mathrm{Rag}^{-/-} \mathrm{II2 \textrm {rg } ^ { - / - }}$ host mice given no cells (left) or given intravenous transfer of $1 \times 10^{5} \mathrm{ST}^{+} \mathrm{IL}-18 \mathrm{Ra}{ }^{-} \mathrm{ILC} 2$ cells sorted from the lungs of GFP reporter mice treated intranasally with IL-33, along with $30 \times 10^{6}$ splenocytes and $3 \times 10^{6}$ to $4 \times 10^{6} \mathrm{GFP}^{-} \mathrm{T}$ cells and NK cells derived from the lungs of C57BL/6 mice (middle and right), followed by mock infection of host mice (left and middle) or infection of host mice with influenza virus (right) $12 \mathrm{~h}$ after cell transfer. Numbers adjacent to outlined areas indicate percent $\mathrm{Lin}^{-} \mathrm{GFP}^{+}$ cells. (b) Flow cytometry analyzing the expression of CD25, CD90 and ST2 by transferred $\mathrm{Lin}^{-} \mathrm{GFP}^{+}$ILCs in influenza-virus-infected host mice as in a. (c-e) Flow cytometry of adoptively transferred GFP $^{+}$ILCs in host mice as in a at day 7 after infection. Numbers adjacent to outlined areas indicate percent GATA-3+ ILCs (d), IL-18Ra ${ }^{+}$ILCs (e) or IL-12R $\beta 2^{+}$ILCs (f). (f-h) MFI of GATA-3 (f), IL-18Ra (g) and IL-12R $\beta 2$ (h) on adoptively transferred GFP ${ }^{+}$ILCs from mice as in a at day 7 after infection. Each symbol represents an individual mouse; small horizontal lines indicate the mean ( \pm s.e.m.). (i) Microscopy of a lung lobe from a mouse as in a $6 \mathrm{~d}$ after infection with influenza virus, showing $\mathrm{GFP}^{+}$ILCs (brown) clustering near influenza-virus-positive cells (green) in localized areas of inflammation (*). (j) Double IHC for GFP+ ILCs (brown) and influenza virus (green), showing clustering of ILCs in infected bronchioles ( $\mathrm{Inf} \mathrm{Br}$ ) and alveoli (Inf Alv) (right) and scattered cells (arrowheads) in uninfected bronchioles (Br) and alveoli (Alv) (left). Inset (bottom left), $5 \times$ enlargement of an area in the main image. Scale bars, $1.2 \mathrm{~mm}$ (i) or $100 \mu \mathrm{m}(\mathbf{j}) . * P<0.01$ and $* * P<0.001$ (Mann-Whitney Wilcoxon test). Data are 
representative of three independent experiments with four mice (mock infection) or five mice (viral infection) (mean in $\mathbf{a}, \mathbf{c}-\mathbf{e})$. 
a

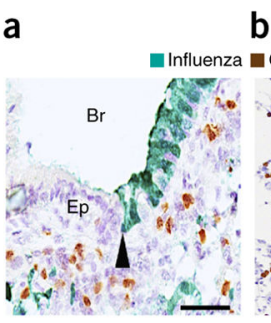

f

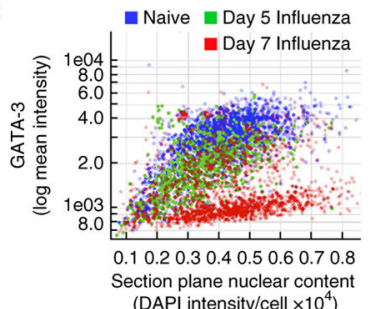

c - $1118 \mathrm{mRNA}$ Ep
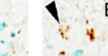

Ep

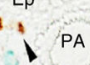

d $=\begin{aligned} & \text { GFP IHC } \\ & \text { III12 mRNA }\end{aligned}$

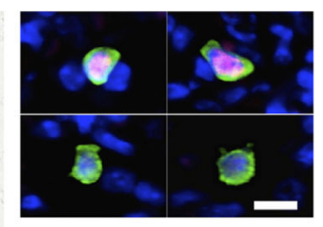

h

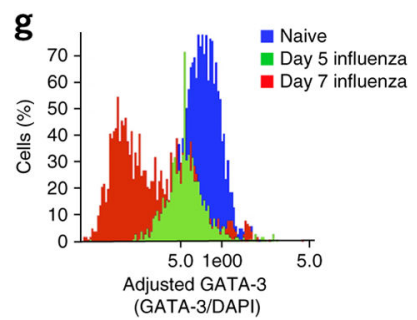

e i
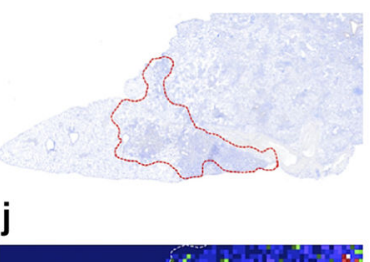

Figure 5.

ILC2 cells cluster in areas associated with viral replication and type 1 cytokine production.

(a) Microscopy of lung tissue from an influenza-virus-infected host mouse as in Figure 4a, showing $\mathrm{GFP}^{+}$cells (brown) among virus-infected airway epithelial cells (Ep) (green). (b) Perivascular accumulation of $\mathrm{GFP}^{+}$cells adjacent to a pulmonary vessel (PV) in an infected lung region of a mouse as in a. (c,d) Combined IHC and in situ hybridization of lung tissue from a mouse as in a, showing spatial association between $\mathrm{GFP}^{+}$ILCs (green) and cells (brown; arrowheads) expressing $I 118$ mRNA (c) or $I 112$ mRNA (d). PA, pulmonary artery. (e) Images of $\mathrm{GFP}^{+}$cells (green), DAPI-stained nuclei (blue) and GATA-3 immunoreactivity (red-pink) of lungs from host mice as in Figure 4a, showing GATA- ${ }^{\text {hi }}$ ILCs (top row) in lungs from an uninfected control mouse (left) and in uninfected regions of lungs from an influenza-virus-infected mouse at day 7 after infection (right), or GATA- $3^{\text {lo }}$ ILCs (bottom row) in lungs from influenza-virus-infected mice at $5 \mathrm{~d}$ (left) or $6 \mathrm{~d}$ (right) after infection. (f) GATA-3 expression in individual $\mathrm{GFP}^{+}$cells from mice as in Figure 4a (key), plotted against nuclear content (mean intensity of DAPI in regions of interest). (g) Frequency of pooled $\mathrm{GFP}^{+}$cells in mice as in $\mathbf{f}$, plotted against GATA-3 expression (adjusted relative to DAPI staining (GATA-3/DAPI)). (h) GATA-3 expression in ILCs from host mice as in as in Figure $4 \mathrm{a}$, at $6 \mathrm{~d}$ after infection. (i) Microscopy of a hematoxylin-stained lung from a host mouse as in Figure $4 \mathrm{a}$ at $5 \mathrm{~d}$ after infection with influenza virus, showing a patchy area of enhanced cellularity (red outline), indicative of infection. ( $\mathbf{j}$ ) Heat map of a section as in $\mathbf{i}$, stained for influenza A virus to assess viral density (to confirm viral infection of area outlined in i). (k) Spatial distribution of $\mathrm{GFP}^{+}$ILCs as in $\mathbf{i}$; each symbol represent an individual $\mathrm{GFP}^{+}$cell (colors, key). Scale bars, $40 \mu \mathrm{m}(\mathbf{a}, \mathbf{b})$ or $12 \mu \mathrm{m}(\mathbf{e})$. Original magnification, $\times 40(\mathbf{c}, \mathbf{d})$ or $\times 10$ (i-k). $* P<0.001$ and $* * P<0.001$ (Mann-Whitney Wilcoxon test). Data are representative of three experiments (a-e,i-k), two experiments with 700-1,700 cells per condition (f, $\mathbf{g})$ or two independent experiments with six mice per group (h; mean + s.e.m.). 
a
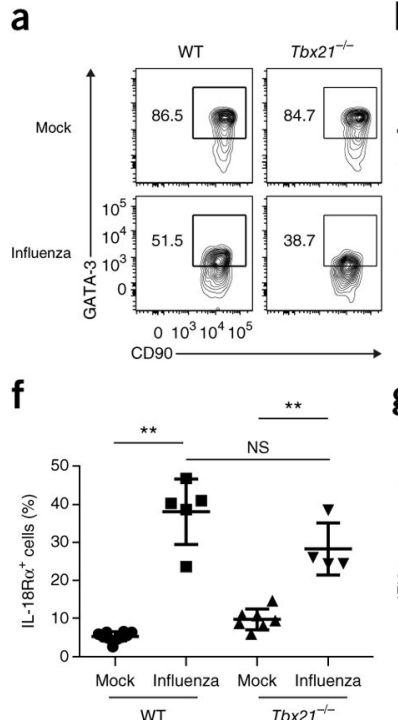

b

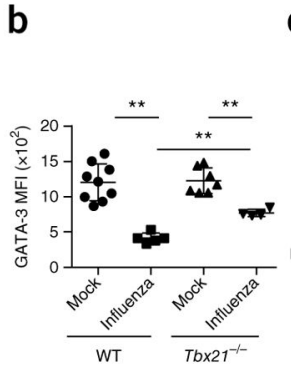

g
C

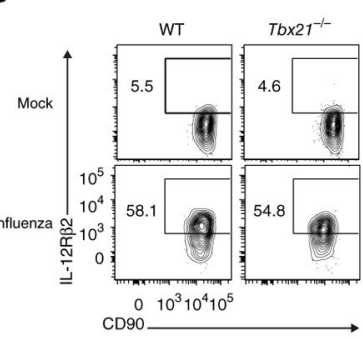

d

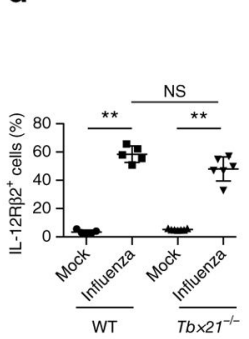

$$
\mathbf{h}^{10}
$$

e
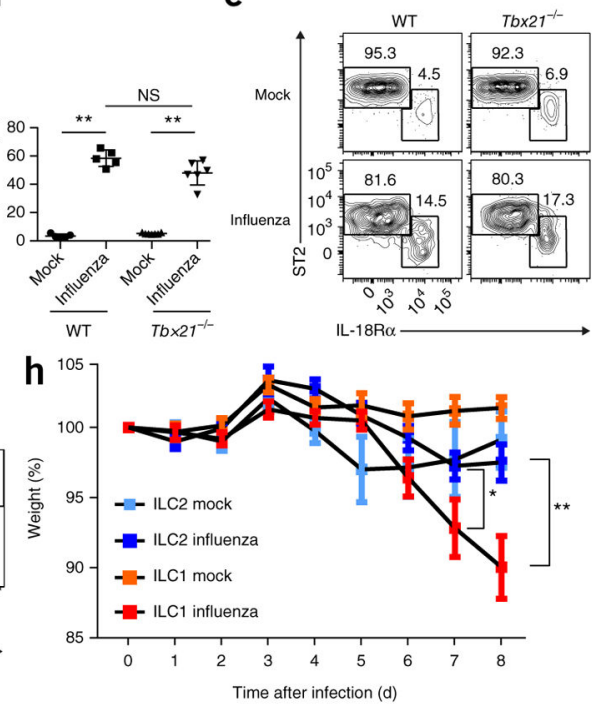
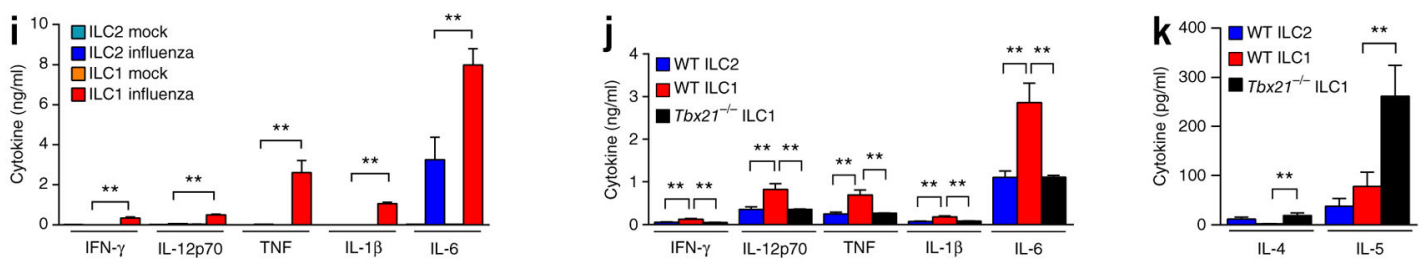

Figure 6.

ILC1 cells augment antiviral immunity in a T-bet-dependent manner. (a-f) Flow cytometry of lung-resident ILCs from C57BL/6 wild-type (WT) and $T b \times 21^{-1-}$ mice at day 7 after mock infection or challenge with influenza virus strain PR8. Numbers adjacent to outlined areas $(\mathbf{a}, \mathbf{c}, \mathbf{e})$ indicate percent GATA-3 ${ }^{+}$ILCs (a), IL-12R $\beta 2^{+}$ILCs (c), or ST2 ${ }^{+} \mathrm{IL}-18 \mathrm{Ra}{ }^{-}$cells (top left) or ST2-IL-18Ra ${ }^{+}$cells (top right) (e). (b,d,f) MFI of GATA-3 (b), or frequency of IL-12R $\beta 2^{+}$cells $(\mathbf{d})$ or IL-18Ra ${ }^{+}$cells $(\mathbf{f})$. Each symbol $(\mathbf{b}, \mathbf{d}, \mathbf{f})$ represents an individual mouse; small horizontal lines indicate the mean ( \pm s.e.m.). (g) Flow cytometry of ILCs enriched from C57BL/6 wild-type and $T b \times 21^{-1-}$ mice at day 7 after mock infection (left) or infection with influenza virus (right). Numbers adjacent to outlined areas indicate percent IFN- $\gamma^{+}$ILCs. (h,i) Weight (h) and cytokine concentrations in BAL fluid (i) of $\mathrm{Rag}^{-/-}$II $2 \mathrm{rg}^{-1-}$ host mice given ILC2 or ILC1 cells purified from mice treated with IL-33 (ILC2) or IL-33 plus IL-12 plus IL-18 (ILC1), assessed $2 \mathrm{~d}$ after mock infection of host mice or infection of host mice with influenza virus (key). (j,k) Expression of cytokines in BAL fluid of $R a g 2^{-1-} I I 2 \mathrm{rg}^{-/-}$host mice reconstituted with ILC2 or ILC1 cells purified from C57BL/6 wild-type or $T b \times 21^{-1-}$ mice (key), assessed $2 \mathrm{~d}$ after infection of host mice with influenza virus. $* P<0.01$ and $* * P<0.001$ (Mann-Whitney Wilcoxon test). Data are representative of two independent experiments with five mice per group (C57BL/6 wildtype), six mice per group ( $T b \times 21^{-1-}$ infected) or seven mice per group $\left(\mathrm{Tb} \times 21^{-/-}\right.$mock infected) (a-g) or two independent experiments with four mice per group (ILC2 reconstitution; ILC1 reconstitution with mock infection) or five mice per group (ILC1 reconstitution with viral infection) (h,i) or are from one experiment with seven mice per group $(\mathbf{j}, \mathbf{k})$. 

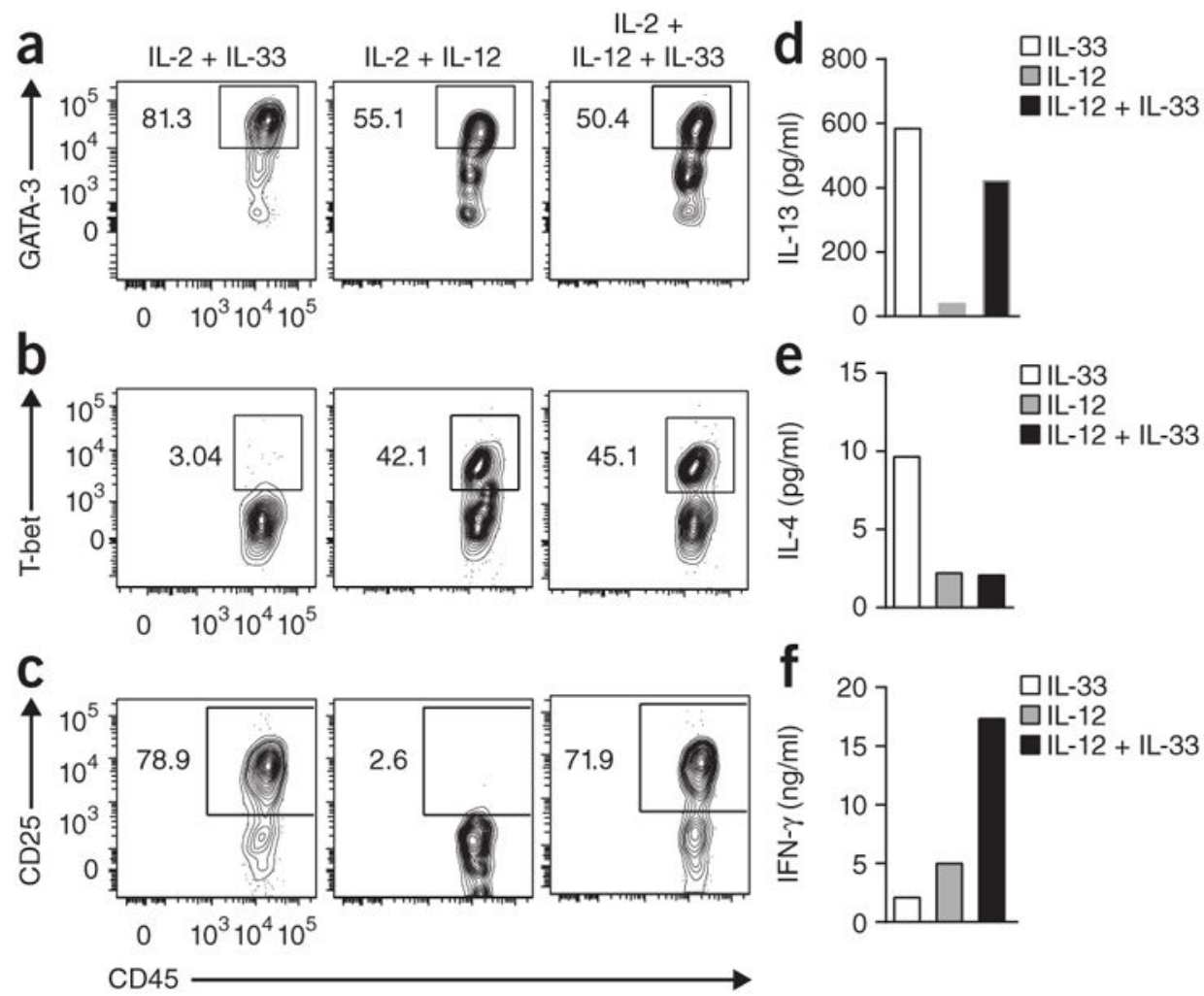

Figure 7.

IL-12 induces plasticity in human ILC2 cells. (a-c) Flow cytometry of ILC2 cells sorted from the peripheral blood of healthy human donors and cultured for $5 \mathrm{~d}$ with IL-2 plus IL-33 or IL-12 or with IL-2 plus IL-33 and IL-12 (above plots). Numbers adjacent to outlined areas indicate percent GATA-3 ${ }^{+}$ILCs (a), T-bet ${ }^{+}$ILCs (b) or CD25 $5^{+}$ILCs (c). (d-f) Concentration of IL-13 (d), IL-4 (e) or IFN- $\gamma$ (f) in culture supernatants of cells as in a-c. Data are representative of four independent experiments with cells pooled from three to four donors in each. 
a

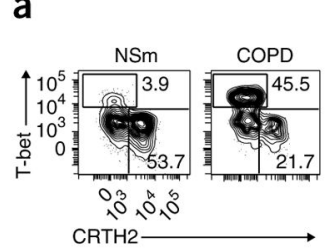

f

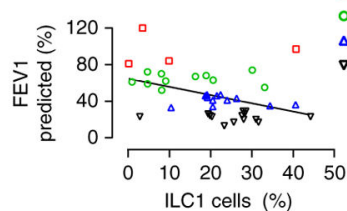

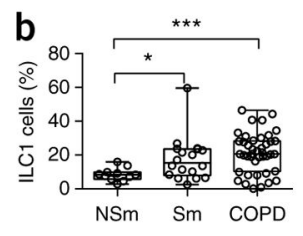
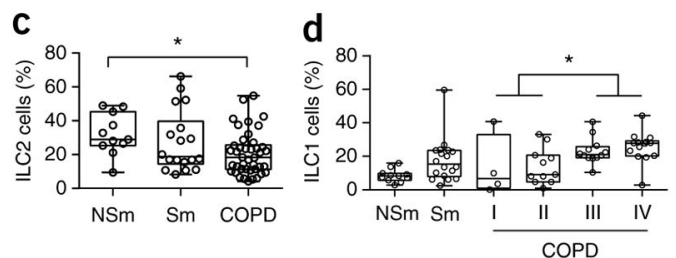

h

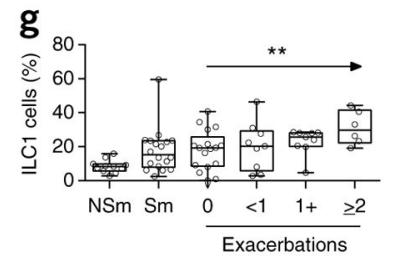

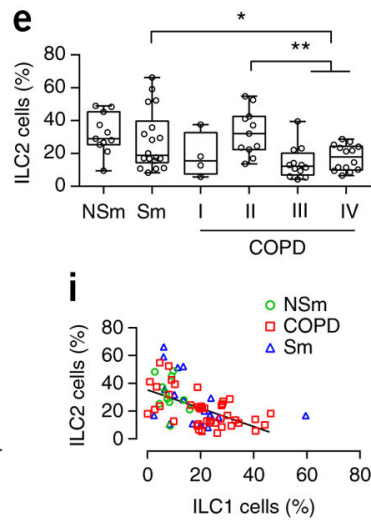

Figure 8.

ILCs are substantially altered in COPD. (a) Flow cytometry of ILCs in peripheral blood of a non-smoking control subject (NSm) (left) and a patient with COPD (right). Numbers adjacent to or in outlined areas indicate percent T-bet ${ }^{+} \mathrm{CRTH} 2^{-}$cells (top left) or Tbet $^{-} \mathrm{CRTH} 2^{+}$cells (bottom right). (b,c) Frequency of circulating ILC1 (T-bet ${ }^{+}$) cells $(\mathbf{b})$ or ILC2 $\left(\mathrm{CRTH}^{+}\right)$cells (c) in non-smoking (healthy) control subjects (NSm), smoking control subjects (Sm) and patients with COPD (COPD). (d,e) Frequency of circulating ILC1 cells (d) and cells ILC2 (e) in non-smoking (healthy) control subjects, smoking control subjects and patients with COPD, stratified by GOLD status (horizontal axes). (f) Correlation between frequency of ILC1 cells and FEV1 in patients with COPD, stratified by GOLD status (key). $R=-0.4162$. (g,h) Frequency of circulating ILC1 cells (g) and ILC2 cells (h) in non-smoking (healthy) control subjects, smoking control subjects and patients with COPD, stratified by exacerbation frequency (average exacerbations per year; horizontal axes). (i) Correlation between frequency of circulating ILC2 cells and that of ILC1 cells in nonsmoking (healthy) control subjects, smoking control subjects and patients with COPD. $R=$ -0.0553. $* P<0.05, * * P<0.01$ and $* * * P<0.001$ one-way mixed effect ANOVA (b, $\mathbf{c}, \mathbf{d}, \mathbf{e}$, $\mathbf{g}, \mathbf{h})$ Pearson correlation coefficient $(\mathbf{f}, \mathbf{i})$. Data are from one experiment $(\mathbf{a})$ or one experiment with $n=11$ donors (non-smoking control), $n=18$ donors (smoking control) or $n$ $=42$ donors $(\mathrm{COPD}))(\mathbf{b}, \mathbf{c}, \mathbf{i}), n=11$ donors (non-smoking control), $n=18$ donors (smoking control), $n=4$ donors (COPD GOLD I), $n=11$ donors (COPD GOLD II), $n=12$ donors (COPD GOLD III) or $n=14$ donors (COPD GOLD IV) (d-f), or $n=11$ donors (nonsmoking control), $n=18$ donors (smoking control), $n=17$ (COPD, no exacerbations), $n=9$ (COPD, one exacerbation), $n=9$ (COPD, more than one exacerbation) or $n=6$ (COPD, two or more exacerbations) (g,h). 